\title{
Eigenstate-specific temperatures in two-level paramagnetic spin lattices
}

Mark B. Masthay, Calley N. Eads, Amber N. Johnson, Robert G. Keil, Philip Miller, Ross E. Jones, Joe D. Mashburn, and Harry B. Fannin

Citation: The Journal of Chemical Physics 147, 214306 (2017);

View online: https://doi.org/10.1063/1.5012914

View Table of Contents: http://aip.scitation.org/toc/jcp/147/21

Published by the American Institute of Physics

\section{Articles you may be interested in}

The gas phase structure of $\alpha$-pinene, a main biogenic volatile organic compound

The Journal of Chemical Physics 147, 214305 (2017); 10.1063/1.5003726

Analytical energy gradients for explicitly correlated wave functions. I. Explicitly correlated second-order Møller-Plesset perturbation theory

The Journal of Chemical Physics 147, 214101 (2017); 10.1063/1.5003065

On the non-stationary generalized Langevin equation

The Journal of Chemical Physics 147, 214110 (2017); 10.1063/1.5006980

Quantum interference in multi-branched molecules: The exact transfer matrix solutions

The Journal of Chemical Physics 147, 214115 (2017); 10.1063/1.4989872

Perspective: Highly stable vapor-deposited glasses

The Journal of Chemical Physics 147, 210901 (2017); 10.1063/1.5006265

Nuclear spin/parity dependent spectroscopy and predissociation dynamics in $\mathrm{vOH}=2 \leftarrow 0$ overtone excited $\mathrm{Ne}-\mathrm{H}_{2} \mathrm{O}$ clusters: Theory and experiment

The Journal of Chemical Physics 147, 214304 (2017); 10.1063/1.5001335

\section{AIP $\mid$ The Journal of Chemical Physics}




\title{
Eigenstate-specific temperatures in two-level paramagnetic spin lattices
}

\author{
Mark B. Masthay, ${ }^{1, \text { a) }}$ Calley N. Eads ${ }^{2}$ Amber N. Johnson, ${ }^{1}$ Robert G. Keil, ${ }^{1}$ Philip Miller, ${ }^{3}$ \\ Ross E. Jones, ${ }^{3}$ Joe D. Mashburn, ${ }^{4}$ and Harry B. Fannin ${ }^{3}$ \\ ${ }^{1}$ Department of Chemistry, University of Dayton, 300 College Park, Dayton, Ohio 45469-2357, USA \\ ${ }^{2}$ Department of Chemistry and Biochemistry, The University of Arizona, P.O. Box 2100411306 East University \\ Boulevard, Tucson, Arizona 85721-0041, USA \\ ${ }^{3}$ Department of Chemistry, Murray State University, 1201 Jesse D. Jones Hall, Murray, Kentucky 42071, USA \\ ${ }^{4}$ Department of Mathematics, University of Dayton, 300 College Park, Dayton, Ohio 45469-2316, USA
}

(Received 20 June 2017; accepted 7 November 2017; published online 5 December 2017)

\begin{abstract}
Increasing interest in the thermodynamics of small and/or isolated systems, in combination with recent observations of negative temperatures of atoms in ultracold optical lattices, has stimulated the need for estimating the conventional, canonical temperature $T_{c}^{c o n v}$ of systems in equilibrium with heat baths using eigenstate-specific temperatures (ESTs). Four distinct ESTs-continuous canonical, discrete canonical, continuous microcanonical, and discrete microcanonical-are accordingly derived for two-level paramagnetic spin lattices (PSLs) in external magnetic fields. At large $N$, the four ESTs are intensive, equal to $T_{c}^{c o n v}$, and obey all four laws of thermodynamics. In contrast, for $N$ $<1000$, the ESTs of most PSL eigenstates are non-intensive, differ from $T_{c}^{c o n v}$, and violate each of the thermodynamic laws. Hence, in spite of their similarities to $T_{c}^{c o n v}$ at large $N$, the ESTs are not true thermodynamic temperatures. Even so, each of the ESTs manifests a unique functional dependence on energy which clearly specifies the magnitude and direction of their deviation from $T_{c}^{\text {conv }}$; the ESTs are thus good temperature estimators for small PSLs. The thermodynamic uncertainty relation is obeyed only by the ESTs of small canonical PSLs; it is violated by large canonical PSLs and by microcanonical PSLs of any size. The ESTs of population-inverted eigenstates are negative (positive) when calculated using Boltzmann (Gibbs) entropies; the thermodynamic implications of these entropically induced differences in sign are discussed in light of adiabatic invariance of the entropies. Potential applications of the four ESTs to nanothermometers and to systems with long-range interactions are discussed. Published by AIP Publishing. https://doi.org/10.1063/1.5012914
\end{abstract}

\section{INTRODUCTION}

Though temperature is a ubiquitous concept in the physical and biological sciences, its nature and definition have become subjects of fresh debate in the last three decades in two notable contexts: (1) the Feshbach ${ }^{1}-$ Kittel $^{2}-$ Mandelbrot $^{3}$ (FKM) debate regarding the differences between thermodynamic temperatures and the so-called "effective temperatures" 2 or "temperature estimators" 3 for systems in contact with small heat baths ${ }^{2}$ and (2) the debate over the thermodynamic legitimacy of negative temperatures in systems with bounded energy spectra, ${ }^{4-33}$ which was recently reignited ${ }^{4-6,31}$ by the realization of negative temperatures in optical lattices. ${ }^{28-32}$ The $\mathrm{FKM}^{1-3,34-36}$ and negative temperature ${ }^{4-6,27,31}$ debates both address issues central to a number of important topics: (1) the thermodynamics of small systems ${ }^{1-3,34}$ (2) the thermodynamics of isolated systems; ${ }^{12,34,35,37-59}$ (3) the thermodynamic uncertainty relation (TUR); ${ }^{1-3,34-36}$ and (4) quantum thermodynamics, ${ }^{40,60}$ in which the temperatures of individual quantum eigenstates, hereafter designated eigenstate-specific temperatures (ESTs), apply.

\footnotetext{
a)Author to whom correspondence should be addressed: mmasthay1@ udayton.edu. Telephone: (937) 229-2631. Fax: (937) 229-2635.
}

In the present study small, two-level paramagnetic spin lattices (PSLs) ${ }^{7,9,11-18,36,61-66}$ are used to address two questions raised in the $\mathrm{FKM}^{1-3}$ and negative temperature ${ }^{4-25}$ debates: (1) "Are the ESTs of PSLs true thermodynamic temperatures or merely good temperature estimators?" and (2) "Are the negative ESTs of population-inverted PSLs thermodynamically legitimate?" We address these questions by characterizing the size- and energy-dependence of four distinct ESTs: (a) continuous and (b) discrete canonical ESTs, which apply to PSLs in equilibrium with heat baths, and (c) continuous and (d) discrete microcanonical ESTs, which apply to isolated PSLs.

The four ESTs share much in common with the conventional canonical temperature $T_{c}^{\text {conv }}$, which is defined as (1) a parameter which is equal to the temperature $T_{\text {bath }}$ of the system's heat bath ${ }^{2,67}$ and (2) the continuous rate of change $\left.\left(\partial U / \partial S_{c}[U]\right)_{N, V}\right|_{U^{*}}$ of $U$ with respect to the canonical entropy $S_{c}(U)$ evaluated at the most-probable energy $U^{*}$. The continuous canonical and continuous microcanonical ESTs are equal to the continuous (i.e., instantaneous) rates of change of $U$ with respect to $S_{c}(U)$ and the microcanonical entropy $S_{\mu}(U)$ for the initial eigenstates $[N-j, j]$ in transitions between adjacent PSL eigenstates with spin quantum numbers $j$ and $j+1$ : $T_{c}^{j \rightarrow j+1}=\left.\left(\partial U / \partial S_{c}[U]\right)_{N, V}\right|_{U_{j}}$ and $T_{\mu}^{j \rightarrow j+1}=\left.\left(\partial U / \partial S_{\mu}[U]\right)_{N, V}\right|_{U_{j}}$. The discrete canonical and discrete microcanonical ESTs 
$T_{d c}^{j \rightarrow j+1}=\left.\left(\Delta U / \Delta S_{c}\right)_{N, V}\right|_{U_{j}}$ and $T_{d \mu}^{j \rightarrow j+1}=\left.\left(\Delta U / \Delta S_{\mu}\right)_{N, V}\right|_{U_{j}}$ are the discrete analogs of the continuous ESTs.

As their name indicates, the ESTs are eigenstate-specific; they are equal to the temperatures of specific, individual eigenstates. They thus constitute a distinct contrast to $T_{c}^{c o n v}$ $=T_{c}^{\bar{j} \rightarrow \bar{j}+1}$, which is eigenstate-nonspecific ${ }^{11,40,68}$ because $\bar{j}$ is equal to the (typically non-integer) average value of $j$ over a Boltzmann distribution of eigenstates. Even so, ESTs can provide meaningful estimates of $T_{c}^{c o n v}$ in PSLs, particularly when the population distribution is dominated by a single eigenstate (i.e., when $\bar{j}=j$ ), as occurs for microcanonical (i.e., thermally isolated) PSLs, ${ }^{3,69}$ PSLs at low temperatures, large PSLs in the $N \rightarrow \infty$, thermodynamic limit (TDL), ${ }^{42,70-73}$ and PSLs subjected to repetitive magnetization measurements. ${ }^{36,68,74-78}$

This paper is organized as follows. Background materials are provided in Secs. II A and II B. $T_{c}^{c o n v}$ and the four ESTs are derived in Secs. II C and II D. The general properties of the ESTs are detailed in Sec. III A. The spin-permutation antisymmetries (SPAs) characteristic of positive and negative temperature eigenstates are detailed in Sec. III B and the supplementary material. The size- and energy-dependency of the ESTs-with special emphasis on their functional dependence on the spindown mole fraction $X_{j}=j / N$ - are detailed in Sec. III C and the supplementary material. We then detail the adherence of the ESTs to the four laws of thermodynamics (Sec. III D), the relationships of the ESTs to Boltzmann distributions and the TUR, ${ }^{34,35}$ the relationships of the ESTs to temperaturedependent system energy levels (TDSELs) ${ }^{33}$ (Sec. III E), and the FKM debate (Sec. III F). ${ }^{1-3,34-36}$ The implications of the Boltzmann and Gibbs ESTs for the negative temperature debate $^{4-6,27,31}$ are detailed in Sec. III G. We detail the implications of the ESTs for experimental temperature measurements in PSLs $7,13-17,22,54,63-65,79-89$ (Sec. III H), the potential utility of ESTs in nanothermometry ${ }^{60,83,84,90-102}$ (Sec. III I), and potential applications of ESTs to systems with longrange interactions ${ }^{36,60-62,66-68,74,83,84,92-94,103-106}$ (Sec. III J). Finally, a number of issues raised by the present study are detailed in Sec. IV.

\section{THEORETICAL}

\section{A. Properties of two-level paramagnetic spin lattices}

PSLs are fixed arrays of atoms, ions, or molecules with nuclear $7,9,11-17,63-65,79,81,107$ or electron $7,9,11-17,63-65,79,87-89,108-110$ spin. Two-level PSLs ("PSLs" hereafter) result when each site is comprised of a spin- $1 / 2 \hbar$ nucleus, paramagnetic ion, or free radical with a spin-up (magnetic moment $\boldsymbol{\mu}$ parallel to external magnetic field $\boldsymbol{H}$ ) ground state $\uparrow$ with energy

$$
u_{\uparrow}=-\boldsymbol{\mu} \cdot \boldsymbol{H}=-\mu H=-1 / 2 g \beta H=-1 / 2 \varepsilon
$$

and a spin-down ( $\boldsymbol{\mu}$ antiparallel to $\boldsymbol{H}$ ) excited state $\downarrow$ with energy

$$
u_{\downarrow}=-\boldsymbol{\mu} \cdot \boldsymbol{H}=-\mu H=+1 / 2 g \beta H=+1 / 2 \varepsilon,
$$

in which $g$ is the nuclear or electron $g$ factor and $\beta$ is the nuclear or electron (Bohr) magneton. ${ }^{10,111,112}$ For convenience we rescale the single particle energies to $u_{\uparrow}=0$ and $u_{\downarrow}=\varepsilon$.
PSLs are characterized by three important features. First, because their spin sites are localized, the sites are distinguishable so that the Pauli exclusion principle does not apply. ${ }^{12}$ Second, the sites do not interact with each other so that the total internal energy of an $N$-particle PSL eigenstate $\left[N_{\uparrow}\right.$, $\left.N_{\downarrow}\right]=[N-j, j]$ is equal to the sum of the individual particle energies, ${ }^{12}$

$$
U_{P S L}=U_{j}=N_{\downarrow} \varepsilon=j \varepsilon .
$$

Third, because $\varepsilon \propto H$, the energy spectra of PSLs are discrete in high fields but become continuous in the $H \rightarrow 0$ limit.

\section{B. The thermodynamic temperature}

The thermodynamic temperature $T$ is equal to the rate of change of internal energy $U$ with entropy $S$,

$$
T=\left(\frac{\partial U}{\partial S}\right)_{V, N} .
$$

Hence, when the entropy is analytic in $U$, ESTs may be obtained via the expression

$$
\frac{1}{T(U)}=\left(\frac{\partial S[U]}{\partial U}\right)_{V, N} .
$$

\section{The conventional continuous canonical temperature $T_{c}^{c o n v}$}

Using the canonical partition function for a PSL in equilibrium with a heat bath, Kittel ${ }^{11}$ obtained expressions for mean energy

$$
U_{\bar{j}}=\frac{N \varepsilon}{e^{\varepsilon / k T_{c}^{c o n v}}+1}=\bar{j} \varepsilon
$$

and the conventional canonical temperature

$$
\begin{aligned}
T_{c}^{\text {conv }} & =T_{c}^{\bar{j} \rightarrow \bar{j}+1}=\frac{\varepsilon}{k \ln \left(\frac{N \varepsilon-U_{\bar{j}}}{U_{\bar{j}}}\right)}=\frac{\varepsilon}{k \ln \left(\frac{N-\bar{j}}{\bar{j}}\right)} \\
& =\frac{\varepsilon}{k \ln \left(\frac{1-X_{\bar{j}}}{X_{\bar{j}}}\right)}
\end{aligned}
$$

as functions of the average number of spin-down sites $\bar{j}$ and the average spin-down mole fraction $X_{\bar{j}}=\bar{j} / N$. Equation (4) is well behaved for all $\bar{j} \leq N$ provided $\bar{j} \neq 0$ or $N / 2^{113}$ and for all $X_{\bar{j}} \leq 1$ provided $X_{\bar{j}} \neq 0$ or 0.5 . $^{113}$

The canonical entropy $S_{c}\left(U_{\bar{j}}\right)$ is obtained by integrating $d S_{c}\left(U_{\bar{j}}\right)=d U_{\bar{j}} / T_{c}\left(U_{\bar{j}}\right)$ from 0 to $U_{\bar{j}}$, yielding the concavedownward ${ }^{42,70-73}$ expression

$$
\begin{aligned}
& S_{c}\left(U_{\bar{j}}\right)=\int_{0}^{U_{\bar{j}}} d S_{c}\left(U_{\bar{j}}\right)=\frac{k}{\varepsilon} \ln \left[\frac{(N \varepsilon)^{N \varepsilon}}{\left(N \varepsilon-U_{\bar{j}}\right)^{\left(N \varepsilon-U_{\bar{j}}\right)} U_{\bar{j}}}\right] \\
& =k \ln \left[\frac{N^{N}}{(N-\bar{j})^{(N-\bar{j})_{\bar{j}}}}\right]=S_{c}(\bar{j}),{ }^{11}
\end{aligned}
$$

which is well defined provided $0 \leq \bar{j} \leq N .{ }^{113}$ Since it is a function of energy, $S_{c}(U)$ is microcanonical in character; ${ }^{11}$ it can thus also be obtained from the microcanonical partition function. ${ }^{10,112}$ Even so, $S_{c}(U)$ differs from the microcanonical 
entropy $S_{\mu}(U)$ at finite $N$ but converges to $S_{\mu}(U)$ in the TDL $^{42,70-73}$ [compare Eqs. (5) and (7)].

\section{Eigenstate-specific temperatures (ESTs) in PSLs}

For Boltzmann-distributed PSLs, the average number of spin-down lattice sites $\bar{j}=\bar{N}_{\downarrow}$ is typically not an integer $j .^{36,40}$ Consequently, $T_{c}^{c o n v}=T_{c}^{\bar{j} \rightarrow \bar{j}+1}$ is usually not equal to a continuous canonical EST $T_{c}^{j \rightarrow j+1}$. The temperature becomes eigenstate-specific when $\bar{j} \rightarrow j$. This single eigenstate occupancy condition (SEOC) applies in four scenarios: (i) under microcanonical conditions, in which the PSL is in the single eigenstate it occupied at the moment it was isolated from its heat bath;3,69 (ii) at low temperatures, in which only the ground eigenstate is occupied; (iii) in the thermodynamic limit (TDL), in which the Boltzmann distribution is dominated by its mostprobable eigenstate $\left[N-j^{*}, j^{*}\right] ;^{42,70-73}$ and (iv) when PSLs are subjected to repetitive magnetization measurements which narrow the eigenstate distribution. ${ }^{36,68,74-78}$

Four distinct ESTs may be calculated. The continuous microcanonical EST $T_{\mu}^{j \rightarrow j+1}=\left(\partial U_{j} / \partial S_{\mu}\left[U_{j}\right]\right)_{N, V}$ and the continuous canonical EST $T_{c}^{j \rightarrow j+1}=\left(\partial U_{j} / \partial S_{c}\left[U_{j}\right]\right)_{N, V}$ are equal to the derivatives of $U$ with respect to $S_{c}$ and $S_{\mu}$, respectively. They are thus equal to the tangential slopes at the points on the $U$ vs. $S$ profiles corresponding to the initial eigenstates $[N-j, j]$ in $j \rightarrow j+1$ transitions (see Figs. 1 and 2). The discrete microcanonical EST $T_{d \mu}^{j \rightarrow j+1}=\left(\Delta U^{j \rightarrow j+1} / \Delta S_{\mu}^{j \rightarrow j+1}\right)_{N, V}$ and the discrete canonical EST $T_{d c}^{j \rightarrow j+1}=\left(\Delta U^{j \rightarrow j+1} / \Delta S_{c}^{j \rightarrow j+1}\right)_{N, V}$ are equal to the finite difference slopes between the points on the $U$ vs. $S$ profiles corresponding to the initial $[N-j, j]$ and final $[N-j-1, j+1]$ eigenstates in the transitions [see Figs. 2(a)-2(c)].

\section{The continuous microcanonical EST $T_{\mu}^{j \rightarrow j+1}$}

The transition energy between energetically adjacent eigenstates in PSLs is equal to

$$
\Delta U^{j \rightarrow j+1}=\varepsilon=g \beta H .
$$

The microcanonical entropy $S_{\mu}\left(U_{j}\right)$ of $[N-j, j]$ is equal to

$$
\begin{aligned}
S_{\mu}\left(U_{j}\right) & =k \ln \Omega_{j}=k \ln \left(\frac{N !}{[N-j] ! j !}\right) \\
& =k \ln \left(\frac{\Gamma[N+1]}{\Gamma[N-j+1] \Gamma[j+1]}\right),
\end{aligned}
$$

in which the gamma function $\Gamma(j+1)=j$ ! is introduced to make the entropy a continuous function of $j .{ }^{114}$ Taking the derivative with respect to $j$ and inverting the result yields

$$
\begin{aligned}
T_{\mu}^{j \rightarrow j+1} & =\frac{\varepsilon}{k\left(\Psi_{0}[N-j+1]-\Psi_{0}[j+1]\right)} \\
& =\frac{\varepsilon}{k\left(\sum_{p=1}^{N-j} \frac{1}{p}-\sum_{p=1}^{j} \frac{1}{p}\right)},
\end{aligned}
$$

in which $\Psi_{0}(x)$ is the digamma function. ${ }^{68,115}$ Eq. (8a) is well defined for all $0 \leq j \leq N^{113}$ provided $j \neq N / 2$. As a function of
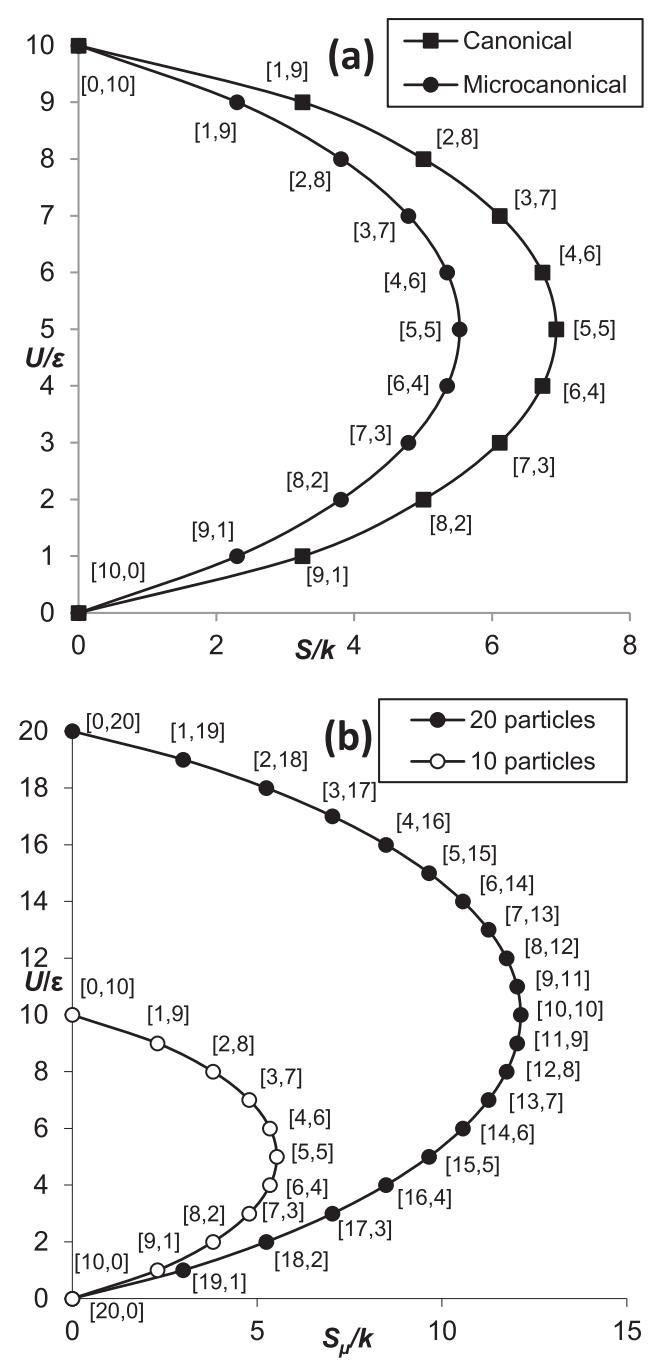

FIG. 1. (a) Internal energy-entropy profiles for $N=10$ PSL under microcanonical and canonical conditions. (b) Internal energy-entropy profiles for microcanonical PSLs with $N=10$ and $N=20$.

the spin-down mole fraction, $T_{\mu}^{j \rightarrow j+1}$ is equal to

$$
\begin{aligned}
T_{\mu}^{j \rightarrow j+1} & =\frac{\varepsilon}{k\left(\Psi_{0}\left[\left(1-X_{j}\right) N+1\right]-\Psi_{0}\left[X_{j} N+1\right]\right)} \\
& =\frac{\varepsilon}{k\left(\sum_{p=1}^{N\left(1-X_{j}\right)} \frac{1}{p}-\sum_{p=1}^{N X_{j}} \frac{1}{p}\right)},
\end{aligned}
$$

which is well behaved for all $0 \leq X_{j} \leq 1^{113}$ provided $X_{j} \neq 0.5$. $T_{\mu}^{j \rightarrow j+1}$ approaches $\mp \infty$ in the limits as $j \rightarrow(N / 2)^{ \pm}$and $X_{j} \rightarrow 0.5^{ \pm}$.

\section{The continuous canonical EST $T_{c}^{j \rightarrow j+1}$}

When $\bar{j}$ is equal to the spin-down quantum number $j$ of an eigenstate $[N-j, j]$, the thermodynamic functions become eigenstate-specific. Under these conditions, eigenstate-specific energy, temperature, and entropy expressions are obtained by substituting $j$ for $\bar{j}$ in Eqs. (3)-(5), yielding

$$
U_{j}=\frac{N \varepsilon}{e^{\varepsilon / k T_{c}}+1}=j \varepsilon,
$$



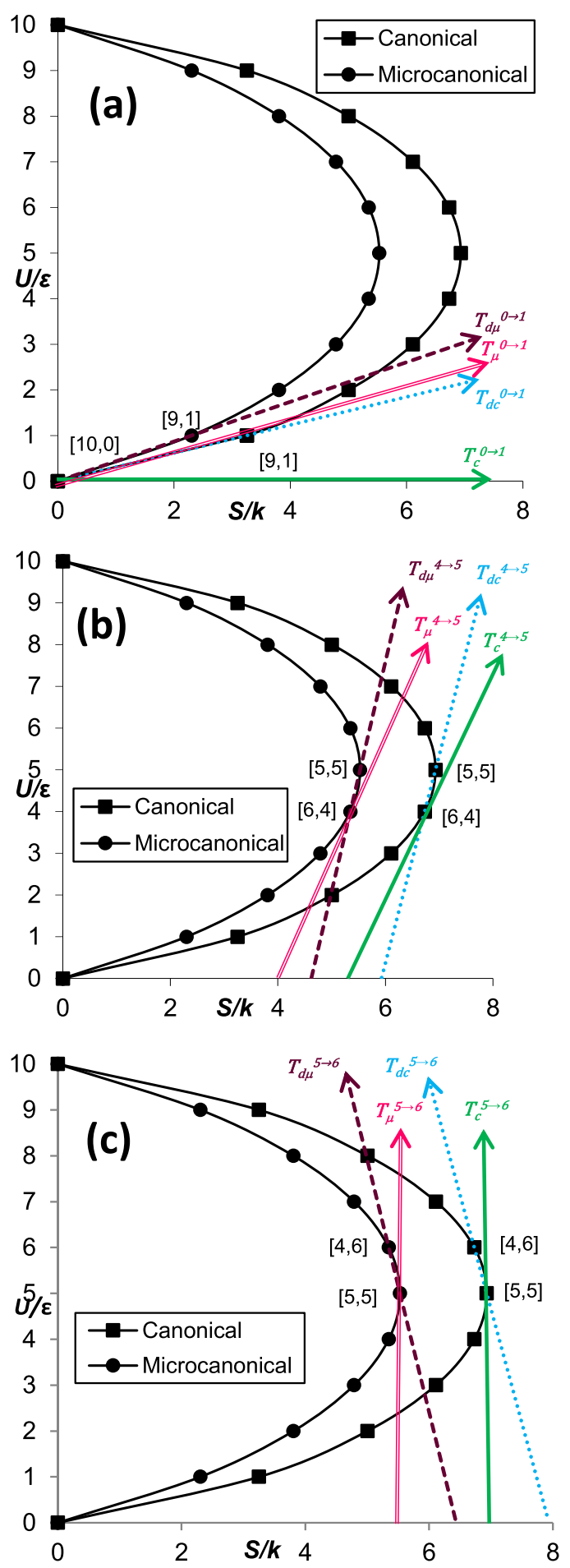

FIG. 2. Continuous microcanonical $\left(T_{\mu}^{j \rightarrow j+1}\right)$, continuous canonical $\left(T_{c}^{j \rightarrow j+1}\right)$, discrete microcanonical $\left(T_{d \mu}^{j \rightarrow j+1}\right)$, and discrete canonical $\left(T_{d c}^{j \rightarrow j+1}\right)$ ESTs of the (a) $[10,0] \rightarrow[9,1]$, (b) $[6,4] \rightarrow[5,5]$, and (c) $[5,5] \rightarrow[4,6]$ transitions in an $N=10$ PSL. $T_{\mu}^{j \rightarrow j+1}$ and $T_{c}^{j \rightarrow j+1}$ are equal to the slopes of the tangents to the profiles at the initial points in the transitions; $T_{d \mu}^{j \rightarrow j+1}$ and $T_{d c}^{j \rightarrow j+1}$ are equal to the finite difference slopes between the initial and final points in the transitions.

$$
T_{c}^{j \rightarrow j+1}=\frac{\varepsilon}{k \ln \left(\frac{N \varepsilon-U_{j}}{U_{j}}\right)}=\frac{\varepsilon}{k \ln \left(\frac{N-j}{j}\right)}=\frac{\varepsilon}{k \ln \left(\frac{1-X_{j}}{X_{j}}\right)},
$$

and the concave-downward ${ }^{42,70-73}$ entropy expression

$$
\begin{aligned}
S_{c}\left(U_{j}\right) & =\frac{k}{\varepsilon} \ln \left[\frac{(N \varepsilon)^{N \varepsilon}}{\left(N \varepsilon-U_{j}\right)^{\left(N \varepsilon-U_{j}\right)} U_{j}^{U_{j}}}\right] \\
& =k \ln \left[\frac{N^{N}}{(N-j)^{(N-j)} j^{j}}\right]=S_{c}(j) .{ }^{11}
\end{aligned}
$$

Equation (10) is well behaved for all $j \leq N$ provided $j \neq 0$ or $N / 2^{113}$ and for all $0 \leq X_{j} \leq 1$ provided $X_{j} \neq 0$ or 0.5 . $^{113}$ $T_{c}^{j \rightarrow j+1}$ approaches zero in the limits as $j \rightarrow 0^{+}$and $X_{j} \rightarrow 0^{+}$; it approaches $\mp \infty$ in the limits as $j \rightarrow(N / 2)^{ \pm}$and $X_{j} \rightarrow 0.5^{ \pm}$.

\section{The discrete microcanonical EST $T_{d \mu}^{j \rightarrow j+1}$} yield

$T_{d \mu}^{j \rightarrow j+1}$ is obtained by combining Eqs. (2a), (6), and (7) to

$$
\begin{aligned}
T_{d \mu}^{j \rightarrow j+1} & =\left(\frac{\Delta U^{j \rightarrow j+1}}{\Delta S_{d \mu}^{j \rightarrow j+1}}\right)_{V, N}=\frac{\varepsilon}{k \ln \left(\frac{N \varepsilon-U_{j}}{U_{j}+\varepsilon}\right)} \\
& =\frac{\varepsilon}{k \ln \left(\frac{N-j}{j+1}\right)}=\frac{\varepsilon}{k \ln \left[\frac{N\left(1-X_{j}\right)}{X_{j} N+1}\right]},
\end{aligned}
$$

which is well behaved for $0 \leq j<N^{113}$ and $j \neq(N-1) / 2$ and for $0 \leq X_{j}<1^{113}$ and $X_{j} \neq 0.5(1-1 / N) . T_{d \mu}^{j \rightarrow j+1}$ approaches $\mp \infty$ in the limits as $j \rightarrow(N-1) / 2^{ \pm}$and $X_{j} \rightarrow 0.5(1-1 / N)^{ \pm}{ }^{116}$

\section{The discrete canonical EST $T_{d c}^{j \rightarrow j+1}$}

$T_{d c}^{j \rightarrow j+1}$ is equal to the finite difference ratio

$$
\begin{aligned}
T_{d c}^{j \rightarrow j+1} & =\frac{\Delta U^{j \rightarrow j+1}}{\Delta S_{c}^{j \rightarrow j+1}}=\frac{\varepsilon}{k \ln \left[\frac{(N-j)^{(N-j)} j}{(N-j-1)^{(N-j-1)}(j+1)^{(j+1)}}\right]} \\
& =\frac{\varepsilon}{k \ln \left[\frac{\left(N\left[1-X_{j}\right]\right)^{(N[1-X j])}\left(N X_{j}\right)^{N X_{j}}}{\left(N\left[1-X_{j}-1 / N\right]\right)^{(N[1-X j-1 / N])}\left(N X_{j}+1\right)^{(N X j+1)}}\right]},
\end{aligned}
$$

in which $\Delta U^{j \rightarrow j+1}$ and $\Delta S_{c}^{j \rightarrow j+1}$ are obtained using Eqs. (6) and (11). Equation (13) is well behaved provided $0 \leq j<N^{113,117}$ and $j \neq(N-1) / 2$ and provided $0 \leq X_{j}<1^{113,117}$ and $X_{j} \neq 0.5$ $(1-1 / N) . T_{d c}^{j \rightarrow j+1}$ approach $\mp \infty$ in the limits as $j \rightarrow(N-1) /$ $2^{ \pm 116}$ and $X_{j} \rightarrow 0.5(1-1 / N)^{ \pm} .{ }^{116}$ Equation (13) is to our knowledge the first derivation of discrete canonical temperatures in any context.

\section{RESULTS AND DISCUSSION}

\section{A. Positive, infinite, and negative absolute temperatures in PSLs}

Conventional absolute (Kelvin) temperatures $T_{c}^{c o n v}$ are (1) positive because the translational entropy of an ideal gas increases monotonically with increasing energy, ${ }^{118}$ (2) finite because for entropically monotonic systems, infinite temperatures occur only in the limit of infinite energy, (3) continuous because the energetic splittings between the translational energy levels of ideal gases are small, ${ }^{118}$ and (4) intensive (i.e., independent of $N$ ) because typical systems are large $\left(N \geq 10^{18}\right)$ and because ideal gas particles are non-interacting. ${ }^{36,60-62,66-68,74,83,84,92-94,103-106}$ In contrast to those of ideal gases, the entropies of PSLs ${ }^{7-9,11-20,63-65,79}$ 
and other energetically bounded systems $\mathrm{s}^{7,21-25,28-32}$ increase with energy for eigenstates with energies between the ground $(U=0)$ and median energy $(U=N \varepsilon / 2)$ but decrease upon further increases in energy. Hence, the ESTs are positive, infinite, and negative for $U_{j}<N \varepsilon / 2, U_{j}=N \varepsilon / 2$, and (populationinverted) $U_{j}>N \varepsilon / 2$ eigenstates, ${ }^{7-9,11-17,20}$ respectively (see Figs. 1 and 2).

Negative spin temperature (i.e., population-inverted) PSL eigenstates are populated under two conditions: (i) upon rapid $180^{\circ}$ rotation of an external magnetic field, which permutes the spin-up and spin-down states; $;^{8,9,12,15}$ and (ii) upon repetitive magnetization measurements. ${ }^{68,74-77}$ Neither of these conditions involves direct thermal heating: Population inversions can be achieved only through non-thermal means. ${ }^{7-9,11-18,21-32,36,61-66}$

\section{B. Spin permutation antisymmetries (SPAs)}

ESTs change sign upon permuting the spin-up and spindown spin sites; that is, they manifest spin permutation antisymmetry. The most significant manifestation of spin permutation antisymmetry is the difference in the $j$ values for which the continuous and discrete ESTs become infinite (see Table I and the supplementary material). ${ }^{119}$

TABLE I. Continuous microcanonical, ${ }^{\mathrm{a}}$ continuous canonical, ${ }^{\mathrm{b}}$ discrete microcanonical, ${ }^{\mathrm{c}}$ and discrete canonical ${ }^{\mathrm{d}}$ ESTs for $j \rightarrow j+1$ transitions ${ }^{\mathrm{e}}$ in two-level, 10-particle PSLs, illustrating differing spin permutation antisymmetries for continuous and discrete ESTs [see Eqs. (B3)-(B10) and (B12)-(B15) in Appendix B in the supplementary material].

\begin{tabular}{lcccc}
\hline \hline Transition $^{\mathrm{e}}[N-j, j]$ & $T_{\mu}^{j \rightarrow j+1}$ & $T_{c}^{j \rightarrow j+1}$ & $T_{d \mu}^{j \rightarrow j+1}$ & $T_{d c}^{j \rightarrow j+1}$ \\
$\rightarrow[N-j-1, j+1]$ & $\left(\frac{\varepsilon}{k}\right)^{\mathrm{a}}$ & $\left(\frac{\varepsilon}{k}\right)^{\mathrm{b}}$ & $\left(\frac{\varepsilon}{k}\right) \mathrm{c}$ & $\left(\frac{\varepsilon}{k}\right) \mathrm{d}$ \\
\hline$[10,0] \rightarrow[9,1]$ & 0.3414 & $0^{\mathrm{f}}$ & 0.4343 & 0.3076 \\
{$[9,1] \rightarrow[8,2]$} & 0.5468 & 0.4551 & 0.6649 & 0.5704 \\
{$[8,2] \rightarrow[7,3]$} & 0.8211 & 0.7213 & 1.019 & 0.9053 \\
{$[7,3] \rightarrow[6,4]$} & 1.316 & 1.180 & 1.787 & 1.609 \\
{$[6,4] \rightarrow[5,5]$} & 2.727 & 2.466 & 5.485 & 4.966 \\
{$[5,5] \rightarrow[4,6]$} & $\pm \infty \mathrm{g}$ & $\pm \infty \mathrm{h}$ & -5.485 & -4.966 \\
{$[4,6] \rightarrow[3,7]$} & -2.727 & -2.466 & -1.787 & -1.609 \\
{$[3,7] \rightarrow[2,8]$} & -1.317 & -1.180 & -1.020 & -0.9053 \\
{$[2,8] \rightarrow[1,9]$} & -0.8211 & -0.7213 & -0.6649 & -0.5704 \\
{$[1,9] \rightarrow[0,10]$} & -0.5468 & -0.4551 & -0.4343 & -0.3076 \\
\hline \hline
\end{tabular}

$\overline{{ }^{a} \text { Continuous microcanonical ESTs of } j \rightarrow j+1 \text { transitions, as given by Eqs. (8a), (8b), }}$ (B3), (B7), and (B12) and calculated using easycalculation.com/digammafunction.php.

${ }^{\mathrm{b}}$ Continuous canonical ESTs of $j \rightarrow j+1$ transitions, as given by Eqs. (10), (B4), (B8), and (B13).

${ }^{\mathrm{c}}$ Discrete microcanonical ESTs of $j \rightarrow j+1$ transitions, as given by Eqs. (12), (B5), (B9), and (B14).

${ }^{\mathrm{d}}$ Discrete canonical ESTs of $j \rightarrow j+1$ transitions, as given by Eqs. (13), (B6), (B10), and (B15).

${ }^{\mathrm{e}} N-j$ and $j$ specify the number of $\operatorname{spin}$-up $\left(U_{\uparrow}=0\right)$ and spin-down $\left(U_{\downarrow}=\varepsilon\right)$ lattice sites, respectively, in a given eigenstate.

${ }^{\mathrm{f}}$ Undefined for $j=0$ but equal to zero in the limit as $j \rightarrow 0^{+}$; i.e., $T_{c}^{0 \rightarrow 1}=\lim _{j \rightarrow 0^{+}} T_{c}^{j \rightarrow j+1}$

$=\lim _{j \rightarrow 0^{+}} \varepsilon / k \ln [(N-j) / j]=0$.

${ }^{\mathrm{g}}$ Undefined for $j=N / 2$, but approaches infinity as $j \rightarrow(N / 2)^{ \pm}$for all $N$ because the denominator of $T_{\mu}^{j \rightarrow j+1}=\varepsilon / k(\Psi[N-j+1]-\Psi[j+1])$ approaches 0 in this limit so that $\lim _{j(N / 2)^{\mp}} T_{\mu}^{j \rightarrow j+1}= \pm \infty$ for all even $N$ and hence is inherently intensive [see Eqs. (B3), (B7), and (B12)].

${ }^{\mathrm{h}}$ Undefined for $j=N / 2$ but approaches infinity as $j \rightarrow(N / 2)^{\mp}$ for all $N$ because the argument of the logarithm in the denominator of $T_{c}^{j \rightarrow j+1}=\varepsilon / k \ln [(N-j) / j]$ approaches 1 in this limit so that $\lim _{j \rightarrow(N / 2)^{\mp}} T_{c}^{j \rightarrow j+1}= \pm \infty$ for all even $N$ and hence is inherently intensive [see Eqs. (10) and (B4)].

\section{Impact of system size and spin-down mole fraction on EST values}

Temperature is typically intensive so that $T=T(U)$. Because the energy of PSLs is extensive, non-intensive temperatures $T(U, N)$ occur when the entropy is non-extensive, in which case the $N$-dependence of the energy numerator in Eq. (2a) is not canceled by a comparable $N$-dependence in the entropy denominator. Non-intensive temperatures occur in small systems, in which finite-size effects ${ }^{36,40,60,68,83,84,92,93}$ are important, and in systems with long-range interactions. ${ }^{36,60-62,66-68,74,83,84,92-94,103-106}$

Because the spin sites in PSLs are non-interacting, it follows that non-intensive ESTs in PSLs originate exclusively from finite-size effects. Although these effects are well known, ${ }^{36,40,60,68,83,84,92,93}$ we report here a previously unrecognized non-intensive temperature behavior which depends functionally on $X_{j}$ in small PSLs. ${ }^{120,121}$

The ESTs may be grouped into triads comprised of sets of three types of energetically adjacent $(j=a N-1, a N$, and $a N$ +1 ) eigenstates: (1) constant- $X_{j}$ eigenstates, for which $X_{j}=a$ is constant with increasing $N$; (2) increasing- $X_{j}$ eigenstates, for which $X_{j}=a-1 / N$ increases with increasing $N$; and (3) decreasing- $X_{j}$ eigenstates, for which $X_{j}=a+1 / N$ decreases with increasing $N$. The constant $0 \leq a \leq 1$ is specific to a given triad.

Although the ESTs in a given triad converge to the common thermodynamic-limiting value $\varepsilon / k \ln \left[\left(1-X_{j}\right) / X_{j}\right]$ $=\varepsilon / k \ln [(1-a) / a]$, the increasing- $X_{j}$, constant- $X_{j}$, and decreasing- $X_{j}$ eigenstates within each triad manifest different functional $N$-dependencies when $N<1000$. This heretofore unreported behavior is demonstrated for continuous canonical ESTs in Eqs. (14a)-(14c) and for the discrete canonical, continuous microcanonical, and discrete microcanonical ESTs in the supplementary material.

The continuous canonical ESTs

$$
T_{c}^{j \rightarrow j+1}=T_{c}^{a N \rightarrow a N+1}=\frac{\varepsilon}{k \ln \left(\frac{1-a}{a}\right)}
$$

of constant- $X_{j}$ eigenstates are converged to $\varepsilon / k \ln [(1-a) / a]$ for all $N$ [see Fig. 3(b)]; these ESTs are inherently intensive. The continuous canonical ESTs

$$
T_{c}^{j \rightarrow j+1}=T_{c}^{a N-1 \rightarrow a N}=\frac{\varepsilon}{k \ln \left(\frac{[1-a] N+1}{a N-1}\right)}
$$

of increasing- $X_{j}$ eigenstates are smaller than $\varepsilon / k \ln [(1-a) / a]$ at small $N$ but ascend to this value as $N \rightarrow \sim 1000$ [see Fig. 4(a)]. In contrast, the continuous canonical ESTs

$$
T_{c}^{j \rightarrow j+1}=T_{c}^{a N+1 \rightarrow a N+2}=\frac{\varepsilon}{k \ln \left(\frac{[1-a] N-1}{a N+1}\right)}
$$

of decreasing- $X_{j}$ eigenstates are larger than $\varepsilon / k \ln [(1-a) / a]$ at small $N$ and descend to this value as $N \rightarrow \sim 1000$ [see Figs. 4(b) and 4(c)]. The continuous microcanonical, discrete microcanonical, and discrete canonical ESTs manifest similar behaviors [see Figs. 3(a), 3(c), and 3(d) and the supplementary material]. These unique small $N$-dependencies suggest that PSLs may prove effective at characterizing the temperatures of systems smaller than those accessible with most currently 

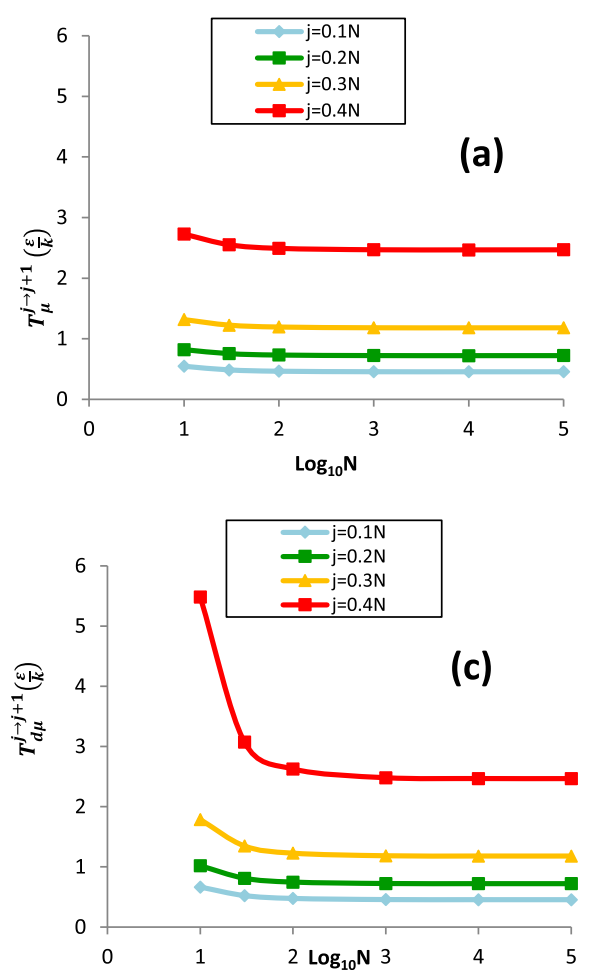
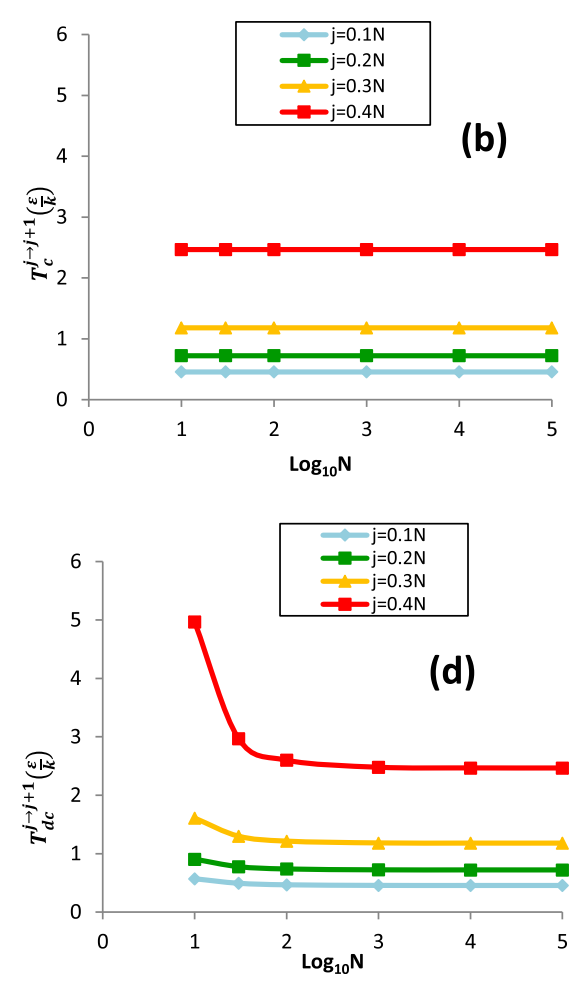

FIG. 3. $N$-dependence of ESTs for $\Delta j$ $=+1$ transitions originating from $X_{j}$ $=a$, constant $-X_{j}$ eigenstates in PSLs: (a) continuous microcanonical, (b) continuous canonical, (c) discrete microcanonical, and (d) discrete canonical ESTs. available nanothermometers ${ }^{60,83,84,90-102}$ (see Sec. III I, Figs. 3 and 4 , and the supplementary material).

\section{The four laws of thermodynamics and the thermodynamic legitimacy of the ESTs}

To be thermodynamically legitimate, ESTs must obey the four laws of thermodynamics. As demonstrated below, this condition applies only when ESTs are intensive.

\section{The zeroth law}

For small $N_{A}$ and $N_{B}$, the ESTs of two PSLs $A$ and $B$ differ if $N_{A} \neq N_{B}$-even when equilibrium (i.e., $X_{j A}=X_{j B}$ ) conditions apply. Under such conditions, $A$ and $B$ can be brought to the same temperature only by adjusting their respective energetic splittings $\varepsilon_{A}$ and $\varepsilon_{B}$. The zeroth law is thus violated by small PSLs; it is obeyed by large PSLs, for which the ESTs are intensive. $^{122}$

\section{The first law}

The first law mandates that the energy change $\Delta U^{j \rightarrow j+1}$ $=C_{V}\left(T_{Y}^{j+1 \rightarrow j+2}-T_{Y}^{j \rightarrow j+1}\right)=\varepsilon$ for $Y=c, d c, \mu, d \mu$. Using the continuous canonical ESTs of positive $\left(X_{j}=0.2\right)$ and negative $\left(X_{j}=0.8\right)$ temperature eigenstates, we find that $\Delta U=\varepsilon$ for both eigenstates when $N \geq 10^{3}$ but that when $N=10, \Delta U$ $=1.411 \varepsilon$ for $X_{j}=0.2$ and $0.819 \varepsilon$ for $X_{j}=0.8$. The continuous canonical ESTs are thus consistent with the first law when $N$ is large but violate this law when $N$ is small; comparable behavior is predicted for the other three ESTs. The first law is thus violated by small PSLs; it is obeyed by large PSLs, for which the ESTs are intensive.

\section{The second law}

When "statistical" changes $d S_{\Omega}=d(k \ln \Omega)$ in the microcanonical entropy are equal to the "thermodynamic" entropy changes $d S_{q / T}=d q / T$, the second law is obeyed. ${ }^{123}$ This property is used here as a criterion for genuine thermodynamic behavior.

As a working system, we assume a $2 N$-particle microcanonical "super-PSL" A + B comprised of two $N$-particle "sub-PSLs" A and B which exchange energy with each other but are otherwise thermally isolated. We further assume that the sub-PSLs are initially in hot $(\mathrm{A}=[0.7 N, 0.3 N])$ and cold $(\mathrm{B}=[0.9 N, 0.1 N])$ eigenstates and that heat flows from $\mathrm{A}$ to $\mathrm{B}$ until both sub-PSLs are in their $[0.8 N, 0.2 N]$ eigenstates. The heat exchange can occur in two ways: (1) a single-step process

$$
([0.7 N, 0.3 N] \stackrel{0.1 N \varepsilon}{\longrightarrow}[0.9 N, 0.1 N]) \Rightarrow([0.8 N, 0.2 N]+[0.8 N, 0.2 N]),
$$

in which $0.1 N$ heat quanta $\varepsilon$ flow instantaneously and isothermally from A to B; and (2) a sequential, multi-step process

$$
\begin{aligned}
& \{[0.7 N, 0.3 N] \stackrel{\varepsilon}{\longrightarrow}[0.9 N, 0.1 N]\} \Rightarrow\{[0.7 N+1,0.3 N-1] \stackrel{\varepsilon}{\longrightarrow} \\
& [0.9 N-1,0.1 N+1]\} \Rightarrow\{[0.7 N+2,0.3 N-2] \stackrel{\varepsilon}{\longrightarrow}[0.9 N-2,0.1 N+2]\} \Rightarrow \cdots \Rightarrow \\
& \{[0.8 N-1,0.2 N+1] \stackrel{\varepsilon}{\longrightarrow}[0.8 N+1,0.2 N-1]\} \Rightarrow\{[0.8 N, 0.2 N])+[0.8 N, 0.2 N]\},
\end{aligned}
$$



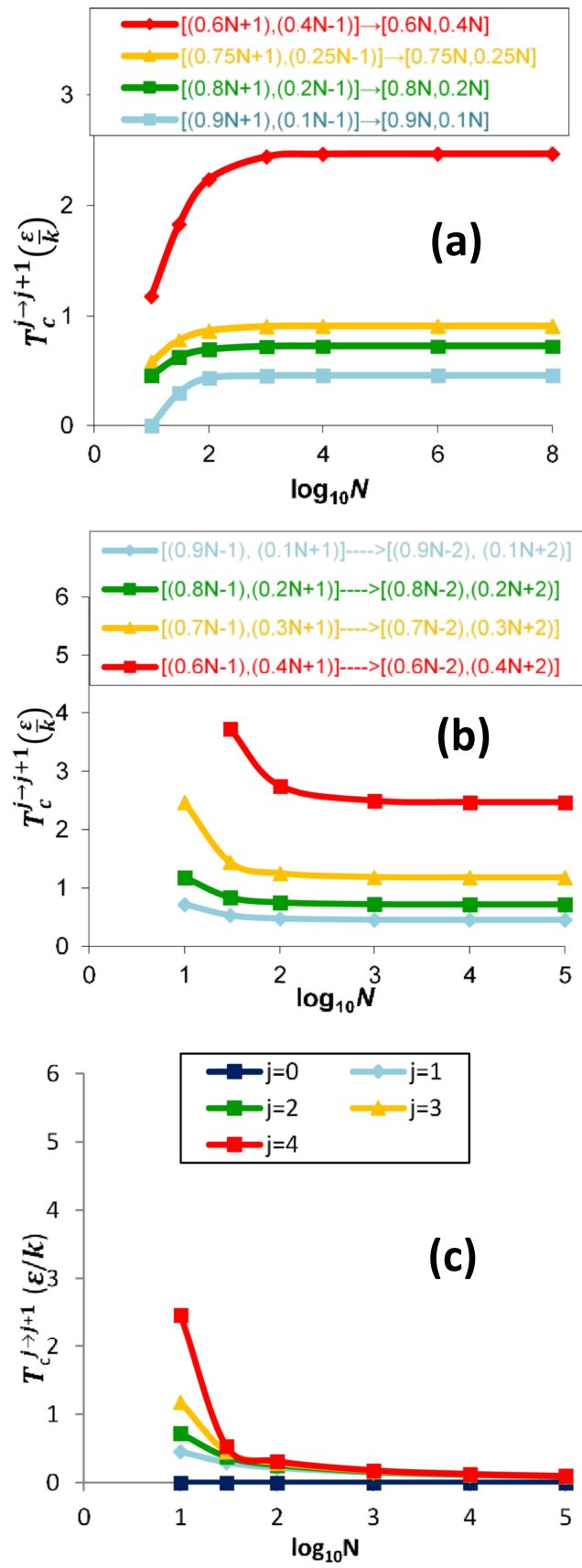

FIG. 4. $N$-dependence of continuous canonical ESTs for $\Delta j=+1$ transitions originating from (a) $X_{j}=a-1 / N$, increasing- $X_{j}$; (b) $X_{j}=a+1 / N$, decreasing$X_{j}$; and (c) $X_{j}=a / N$, decreasing- $X_{j}$ eigenstates in PSLs.

in which $0.1 \mathrm{~N}$ individual heat quanta $\varepsilon$ are successively transferred from A to B and the temperatures of sub-PSLs A and B fall and rise, respectively, with each heat exchange. Since both processes are temperature-independent and share the same initial and final states, the entropy changes are identical for both processes,

$$
\begin{aligned}
\Delta S_{\Omega, \text { single-step }} & =\Delta S_{\Omega, \text { multi-step }}=\Delta S_{\Omega} \\
& =k \ln \left(\Omega_{[0,8 N, 0.2 N]}^{2} / \Omega_{[0.7 N, 0.3 N]} \Omega_{[0.9 N, 0.1 N]}\right) \\
& =(0.0647 k) \times N .
\end{aligned}
$$

The single-step thermodynamic entropy change

$$
\begin{aligned}
\Delta S_{q / T, \text { single-step }}= & \left(-0.1 N \varepsilon / T_{c_{A}}^{0.3 N \rightarrow 0.3 N+1}\right. \\
& \left.+0.1 N \varepsilon / T_{c_{B}}^{0.1 N \rightarrow 0.1 N+1}\right) \\
= & (0.1350 k) \times N
\end{aligned}
$$

is larger than the multi-step thermodynamic entropy change

$$
\begin{aligned}
\Delta S_{q / T, \text { multi-step }}= & \left(-\varepsilon / T_{c_{A}}^{0.3 N \rightarrow 0.3 N+1}+\varepsilon / T_{c_{B}}^{0.1 N \rightarrow 0.1 N+1}\right) \\
& +\left(-\varepsilon / T_{c_{A}}^{0.3 N-1 \rightarrow 0.3 N}+\varepsilon / T_{c_{B}}^{0.1 N+1 \rightarrow 0.1 N+2}\right) \\
& +\left(-\varepsilon / T_{c_{A}}^{0.3 N-2 \rightarrow 0.3 N-1}+\varepsilon / T_{c_{B}}^{0.1 N+2 \rightarrow 0.1 N+3}\right) \\
& +\cdots+\left(-\varepsilon / T_{c_{A}}^{0.2 N+1 \rightarrow 0.2 N}+\varepsilon / T_{c_{B}}^{0.2 N-1 \rightarrow 0.2 N}\right),
\end{aligned}
$$

which at finite $N$ adopts a value lying between those of $\Delta S_{q / T, \text { single-step }}$ and $\Delta S_{\Omega}$. Because they incorporate "excess" entropy contributions originating from the temperature differences between the PSLs, the thermodynamic entropy changes are both larger than $\Delta S_{\Omega}$.

The entropy increases $\Delta S_{q / T}=\left(-\varepsilon / T_{c_{A}}^{j_{A} \rightarrow j_{A}+1}+\varepsilon / T_{c_{B}}^{j_{B} \rightarrow j_{B}+1}\right)$ induced by exchanges of individual heat quanta become smaller as the temperatures of the sub-PSLs converge, i.e., as $j_{A} \rightarrow j_{B} \rightarrow j_{\text {final }}=0.2 N$. Because the number of heat exchanges in which the values of $j_{A}$ and $j_{B}$ are similar increases with increasing $N$, the average excess entropy per exchange is smallest when $N$ is large. Consequently, $\Delta S_{q / T, \text { multi-step }} / \Delta S_{\Omega}$ $\rightarrow 1$ in the TDL: For 10-, 50-, 100-, 500-, and 1000-particle sub-PSLs initially in $X_{j A}=0.3$ and $X_{j B}=0.1$ eigenstates, $\Delta S_{q / T, \text { multi-step }} / \Delta S_{\Omega}=2.081,1.209,1.104,1.020$, and 1.010, respectively. ${ }^{123}$ Hence, $\Delta S_{q / T, \text { multi-step }} \rightarrow \Delta S_{\Omega}$ in the TDL; under these conditions, the second law is obeyed because the ESTs are intensive. In contrast, since both $\Delta S_{q / T}$, single-step and $\Delta S_{\Omega}$ scale with $N, \Delta S_{q / T, \text { single-step }} / \Delta S_{\Omega}=2.086$ is constant for all $N$ so that $\Delta S_{q / T, \text { single-step }}$ does not converge to $\Delta S_{\Omega}$ in the TDL.

Three conclusions can be drawn from these results. First, from the standpoint of entropy, thermodynamics and statistical mechanics are equivalent for PSLs in the TDL because $\Delta S_{q / T, \text { multi-step }} \rightarrow \Delta S_{\Omega}$ as $N$ becomes large. Second, from the standpoint of temperature, statistical mechanics and thermodynamics are equivalent for PSLs in the TDL because the ESTs become intensive at large N. Third, the second law is violated by small PSLs (for which the ESTs are nonintensive) but obeyed by large PSLs (for which the ESTs are intensive).

\section{The third law}

Since a PSL in its ground eigenstates $[N, 0]$ is a perfect spin crystal, the four ESTs should equal zero for this eigenstate when the third law is obeyed. In contrast to this expectation, however, only the continuous canonical EST $T_{c}^{0 \rightarrow 1}$ is equal to $0 \mathrm{~K}$ for all $N ; T_{\mu}^{0 \rightarrow 1}, T_{d \mu}^{0 \rightarrow 1}$, and $T_{d c}^{0 \rightarrow 1}$ are each greater than zero at finite $N$ and approach zero only in the TDL. Hence, when $T_{\mu}^{0 \rightarrow 1}, T_{d \mu}^{0 \rightarrow 1}$, and $T_{d c}^{0 \rightarrow 1}$ are applied, the third law is violated by small PSLs (for which the ESTs are non-intensive) but obeyed by large PSLs (for which the ESTs are intensive; see Table I). ${ }^{6,33,124,125}$ 


\section{E. Relationship of ESTs to Boltzmann distributions and the TUR}

ESTs apply to individual eigenstates regardless of their connection - or lack thereof-to a Boltzmann distribution. This lack of a necessary connection of ESTs to Boltzmann distributions makes their similarities to $T_{c}^{c o n v}$ both intriguing and useful.

$T_{c}^{c o n v}$ is equal to $T_{b a t h}$ only for canonical PSLs which have remained in contact with an infinite bath ${ }^{34-36,67,105,106,126}$ for equilibration time scales $t_{\text {equil }}$ long enough for a Boltzmann distribution to be established. Since a new eigenstate is populated with each PSL $\leftrightarrow$ bath energy exchange, many eigenstates are successively occupied so that the energy and temperature fluctuate during equilibration. ESTs specify the temperature during the brief microcanonical intervals $t_{j}$ $\ll t_{\text {equil }}$ between PSL $\leftrightarrow$ bath energy exchanges.

Three conclusions regarding TUR $\Delta U \Delta(1 / T) \geq k$ follow from the temporal properties of ESTs. First, because the TUR applies only to Boltzmann-distributed systems ${ }^{34-36,67,105,106,126}$ and because the observed energy and temperature values are equal to the energy and the EST of the eigenstate occupied at the time of measurement - and hence change with each successive measurement $-\Delta U$ and $\Delta(1 / T)$ are both non-zero: The energy and temperature measurements both fluctuate so that the TUR is obeyed by small PSLs in equilibrium with infinite baths. ${ }^{34-36,67,105,106,126}$

Second, since large canonical PSLs in equilibrium with infinite baths are dominated by their most-probable eigenstate $\left[N-j^{*}, j^{*}\right],{ }^{42,70-73}$ each consecutive energy and temperature measurement yields the same values $j^{*} \varepsilon$ and $T_{c}^{j * \rightarrow *+1}=T_{c}^{c o n v}$ $=T_{\text {bath }}$. The observed energy and temperature values are thus both non-fluctuating: $\Delta U=\Delta(1 / T)=0$ so that the TUR is violated by large canonical PSLs in equilibrium with infinite baths. ${ }^{34-36,67,105,106,126}$

Third, since they are rigorously microcanonical, all measurements performed on an isolated PSL yield identical energy and temperature values: $\Delta U=0$ (by definition) and $\Delta(1 / T)=\Delta\left(1 / T_{\mu}^{j \rightarrow j+1}\right)=0$ (because the EST is precise). The TUR is thus violated by microcanonical PSLs of any size.

Significantly, this third conclusion disagrees with that of Mandelbrot, ${ }^{3}$ who contended that the TUR applies to a single microcanonical eigenstate "extracted" from a canonical system via thermal isolation. According to Mandelbrot, this eigenstate mysteriously retains the uncertainties in energy and reciprocal temperature of the canonical distribution from which it is extracted. Hence, in agreement with Uffink and van Lith, ${ }^{34}$ we conclude that Mandelbrot's arguments regarding the TUR for microcanonical systems are "counterfactual." "27,128

The three conclusions above are undergirded by a single unifying feature: ESTs are thermodynamically accurate and precise only when they are identical to the thermodynamic temperature. The SEOC applies to rigorously microcanonical systems of any size and effectively to large canonical systems in equilibrium with infinite baths. ${ }^{34-36,67,72,105,106,126}$ Since the ESTs converge in the TDL, differences between the ESTs will not be manifest in scenario (iii); scenarios (i), (ii), and (iv) thus provide the most interesting and important test cases for the utility of ESTs and their applications to the TUR (see Sec. II D).

\section{F. ESTs and the FKM debate}

The FKM debate ${ }^{1-3,34-36}$ was initiated by Feshbach, ${ }^{1}$ who contended that for small systems, $T_{\text {system }}$ can be usefully approximated provided the range of inverse temperature estimates does not exceed the $\Delta(1 / T)$ value specified by the TUR. In response, $\mathrm{Kittel}^{2}$ contended that $T_{\text {system }}$ is defined only for canonical systems - large or small-in equilibrium with large heat baths, in which case $T_{\text {system }}=T_{\text {bath }}$ $=T_{c}^{c o n v}$. Since the large heat capacity of the bath precludes fluctuations in $T_{\text {bath }},{ }^{67,126}$ and since the SEOC is effectively satisfied in large baths, ${ }^{42,70-73}$ both $T_{\text {system }}$ and $T_{\text {bath }}$ are nonfluctuating. ${ }^{2,34,67,105,106,126}$ Mandelbrot $^{3}$ took an intermediate position, in which the actual reciprocal temperature of a canonical system is equal to $T_{b a t h}$ and hence does not fluctuate, but that estimations of the reciprocal temperature obtained using estimators $k \hat{\beta}=\hat{T}^{-1}$ do fluctuate in accord with the TUR: $\Delta U \Delta(k \hat{\beta}) \geq k$.

The ESTs in the present study-which are analogous to Mandelbrot's temperature estimators-are precise regardless of the PSL size. Though they deviate from $T_{b a t h}=T_{c}^{c o n v}$, ESTs provide new insights into many of the issues raised by the FKM debate, ${ }^{1-3,34-36}$ as detailed below.

First, Feshbach was only partially correct: The TUR applies to finite canonical systems, for which energy and temperature measurements fluctuate. Even so, the TUR applies only when Boltzmann statistics are in effect-and this condition prevails only for systems which are in equilibrium with infinite baths. ${ }^{34-36,67,105,106,126}$ Feshbach was thus incorrect to assume that the TUR applies to finite canonical systems in contact with finite baths.

Second, Kittel was likewise only partially correct: The TUR is violated in large canonical systems in equilibrium with infinite baths, ${ }^{34-36,67,105,106,126}$ for which energy and temperature measurements are accurate and precise. He was nevertheless incorrect in concluding that $T_{\text {system }}$ is always equal to $T_{\text {bath }}$ for small canonical systems in equilibrium with infinite baths. $T_{\text {system }}$ and $T_{\text {bath }}$ are not necessarily identical unless the energetic splittings $\varepsilon_{\text {bath }}$ in the bath are effectively continuous; ;,34,67,105,106,126 if $\varepsilon_{\text {bath }}>\varepsilon_{P S L}$, small PSLs in contact with infinite baths can violate both the zeroth law and the TUR. ${ }^{121,129}$

Third, Mandelbrot was also only partially correct: His temperature estimators obey the TUR under canonical conditions provided the bath is infinite, quasi-continuous, and comprised of non-interacting particles. ${ }^{34-36,67,105,106,126}$ Even so, he was incorrect to conclude that his temperature estimators obey the TUR under microcanonical conditions: $3,34,127,128$ The temperatures of microcanonical systems of any size are precise-and hence violate the TUR.

\section{G. Boltzmann and Gibbs ESTs and the negative temperature debate}

Temperature, entropy, and other statistically derived quantities are thermodynamically legitimate when the predictions 
of statistical mechanics concur with thermodynamic measurements. It is generally assumed that temperature is thermodynamically legitimate when it is intensive $e^{130,131}$ and that entropy is thermodynamically legitimate when it is adiabatically invariant (i.e., constant in reversible, adiabatic processes). ${ }^{4-6,27,132}$ Because of the intimate relationship between temperature and entropy, these thermodynamic legitimacy requirements raise the following question: "Are intensive temperatures synonymous with adiabatically invariant entropies?" The answer to this question revolves around the form of the entropy-Boltzmann or Gibbs-used to calculate temperature and is partially addressed by the negative temperature debate, ${ }^{4-6,27,31}$ as detailed below.

The microcanonical Gibbs entropy

$$
S_{\mu G j}=k \ln \sum_{m=0}^{j} \Omega_{m}=k \ln \sum_{m=0}^{j} \frac{N !}{(N-m) ! m !}
$$

for an eigenstate $[N-j, j]$ is equal to the logarithm of the sum of the microcanonical degeneracies of all eigenstates of energy up to and including $[N-j, j]$. It is commonly assumed that the Gibbs entropy is an adiabatic invariant for all $N .^{4-6,27,132,133}$ In contrast, the microcanonical Boltzmann entropies $S_{\mu B j}$ in Eq. (7) are equal to the degeneracy $\Omega_{j}$ of the $[N-j, j]$ eigenstate. It is commonly assumed that $S_{\mu B j}$ is not adiabatically invariant for small $N$ but that it becomes so in the TDL because it converges to $S_{\mu G j}$ as $N$ becomes large. ${ }^{4-6,27}$

Because $S_{\mu B j}$ decreases with increasing energy above the energy median, Boltzmann ESTs of PSLs are negative for population-inverted eigenstates. In contrast, because $S_{\mu G j}$ of PSLs increases monotonically with increasing energy for all $j$, the discrete microcanonical Gibbs ESTs

$$
\begin{aligned}
T_{d \mu G}^{j \rightarrow j+1} & =\left(\frac{\Delta U^{j \rightarrow j+1}}{\Delta S_{G}^{j \rightarrow j+1}}\right)_{V, N} \\
& =\frac{\varepsilon}{k\left(\ln \sum_{m=0}^{j+1} \frac{N !}{(N-m) ! m !}-\ln \sum_{m=0}^{j} \frac{N !}{(N-m) ! m !}\right)}
\end{aligned}
$$

of PSLs are uniformly positive-even for population-inverted eigenstates. ${ }^{4,5,27}$

The thermodynamic legitimacy of Boltzmann entropies and of negative absolute Boltzmann temperatures in PSLs and other energetically bounded systems has recently been challenged, for three reasons: (1) the Gibbs ESTs of PSLs are positive for all eigenstates, whereas the Boltzmann ESTs are negative for population-inverted eigenstates; (2) negative absolute temperatures imply that the Boltzmann populations of population-inverted eigenstates should increase with increasing energy; 8 and (3) Gibbs entropies are commonly believed to be adiabatically invariant, whereas Boltzmann entropies are not. ${ }^{4-6,27}$ Even so, there are strong reasons to believe that negative absolute temperatures are thermodynamically legitimate and that Boltzmann temperatures and entropies are preferable to their Gibbs analogs.

First, the Gibbs ESTs of population-inverted eigenstates grow exponentially with increasing $N^{5}$ and hence manifest no TDL. This super-nonintensive character of the Gibbs ESTs is manifestly non-thermodynamic, ${ }^{4-6,27}$ as it violates both the normal notions of hot and $\operatorname{cold}^{5,31}$ and the zeroth law of thermodynamics. ${ }^{6,31}$ In contrast, the Boltzmann ESTs of population-inverted eigenstates are intensive and thermodynamically legitimate in the TDL (see Sec. III C). ${ }^{5}$ Boltzmann ESTs are thus preferable to their Gibbs analogs in PSLs.

Second, Gibbs entropies are not necessarily adiabatically invariant. Based on the $N$-dependence of the chemical potential, Tavassoli and Montakhab ${ }^{133}$ have recently demonstrated that neither the Gibbs nor the Boltzmann entropies are adiabatic invariants in any system for any value of $N{ }^{5,31,133}$ Hence, neither $S_{G j}$ nor $S_{B j}$ give perfect statistical mechanical-thermodynamic equivalence for thermodynamic observables. It is thus not possible to establish a direct correlation between temperature intensity and entropic adiabatic invariance. Even so, Boltzmann ESTs obey the four laws of thermodynamics when they are intensive (see Sec. III D), whereas the Gibbs ESTs of population-inverted eigenstates are non-thermodynamic. Hence, intensity of temperature is a more important criterion for thermodynamic legitimacy than adiabatic invariance of the entropy.

\section{H. ESTs and earlier experimental temperature measurements with nuclear PSLs}

With few exceptions, $5,54,85$ temperature has been characterized with $T_{c}^{c o n v}$ in previous studies of PSLs. ${ }^{7,9,11-18,63-65,79,89}$ Because PSLs containing more than $10^{21}$ nuclei of each element were used in the earlier studies, ${ }^{13-17,64,65,79,134}$ these PSLs were in the TDL. Hence, the (unreported) nuclear spin ESTs are effectively identical to the (reported) conventional canonical nuclear spin temperatures. ESTs thus provide no new insights into the temperatures of the large PSLs utilized in previous studies; the principal utility of ESTs lies in their application to studies of small PSLs, which may find application in nanothermometry, as detailed below.

\section{ESTs and nanothermometry}

An experimental thermometry setup in which a small PSL-based nanothermometer (PSLnt) can yield accurate measurements of the temperature of a canonical system, provided five conditions are satisfied. First, to ensure that the PSLnt does not perturb the system temperature, the size and heat capacity of the PSLnt must both be small compared to those of the system. Second, to ensure that the bath temperature remains constant during system-bath energy exchanges, the size, and heat capacity of the bath must be large compared to those of the system. Third, the system-bath interaction $\hat{H}_{\text {system-bath }}^{\prime}$ must be large enough to allow the system to equilibrate with the bath but small enough to prevent the bath from perturbing the energies of the system eigenstates, ${ }^{135,136}$ i.e., the bath must be weakly coupled to the system. Fourth, the system-PSLnt interaction $\hat{H}_{\text {system-PSLnt }}^{\prime}$ must be large enough to allow the PSLnt to equilibrate with the system but small enough to preclude changes in the energies of the eigenstates of the system and the PSLnt; ${ }^{135,136}$ i.e., the PSLnt must be weakly coupled to the system. Fifth, to ensure that it measures the temperature of the system exclusively, with minimal inaccuracies induced by the bath, the PSLnt must be effectively uncoupled from the bath; i.e., $\hat{H}_{\text {bath-PSLnt }}^{\prime} \sim 0$. 
Provided the five conditions above are satisfied, the canonical temperatures of both the system and the PSLnt may be characterized following equilibration. Since the net magnetization of a PSLnt is proportional to its energy, the temperature of the PSLnt may be assessed by measuring its net magnetization, ipso facto yielding the system temperature in accord with the zeroth law.

For measuring microcanonical temperatures, we propose a setup utilizing a PSLnt which is similar to the "minimal quantum thermometer" proposed by Dunkel and Hilbert. ${ }^{4}$ Provided the first and fourth conditions above are satisfied, such a setup should yield reliable measurements of the microcanonical Gibbs temperature of a system. Since the microcanonical system is isolated, it will initially be in a single eigenstate. If the PSLnt is first prepared in a (preferably) very low energy state with well-defined initial magnetization by magnetic cooling ${ }^{68,76,77}$ and then brought into contact with the system under constrained conditions in which the combined energy of the system and the PSLnt is constant, then upon equilibration the magnetization of the PSLnt will change. The initial microcanonical temperature of the system may then be inferred by noting the change in the net magnetization of the PSLnt.

Our results regarding the impact of $N_{P S L n t}$ on the four ESTs have two important implications. First, because the four ESTs of a given PSL eigenstate are typically intensive for $N$ $\geq 10^{3}$, our results suggest a minimum temperature intensity size limit of $N \sim 10^{3}$ particles-significantly smaller than the sizes of most existing magnetic nanoparticle $\left(N \geq 10^{6}\right),{ }^{98-102}$ paramagnetic salt $\left(N \geq 10^{20}\right),{ }^{95}$ and optical $\left(N \geq 10^{6}\right)^{96,98}$ nanothermometers. Assuming a PSLnt must be no more than one-tenth the size of its target system, PSLnts could yield reliable intensive temperatures for systems as small as $10^{4}$ particles, potentially resulting in a 1000 -fold reduction in the size of target systems accessible with the smallest currently available magnetic nanothermometers..$^{98-102}$ Second, because the $X_{j}$-dependent deviations of the ESTs from their thermodynamic-limiting values are monotonic with decreasing $N$, reliable-albeit non-intensive-estimates of temperature may be attained with PSLnts containing fewer than 1000 particles (see Sec. III C and Figs. 3 and 4).

\section{J. ESTs in systems with long-range-interactions}

Since the spins of PSLs are non-interacting, it follows that the deviations of the ESTs of small PSLs from their thermodynamic-limiting values originate exclusively from finite-size effects ${ }^{36,40,60,68,83,84,92,93}$ (see Sec. III C). Since inter-particle interactions also give rise to non-intensive temperatures, ${ }^{36,60-62,66-68,74,83,84,92-94,103-106}$ any contrasts between the temperatures of PSLs and those of comparably sized systems with long-range spin-spin interactions will provide new insights into the relative impact of finite-size effects and inter-particle interactions on temperature non-intensity.

\section{CONCLUSIONS AND FUTURE STUDIES}

Four types of eigenstate-specific temperatures-continuous canonical, continuous microcanonical, discrete canonical, and discrete microcanonical-have been derived for two-level paramagnetic spin lattices. To our knowledge, this study constitutes the first detailed application of continuous microcanonical and discrete canonical ESTs to PSLs. ${ }^{137}$

Our results lead us to conclude the following. First, the Boltzmann ESTs of small $\left(N \leq 10^{3}\right)$ PSLs deviate from their thermodynamic-limiting values in previously unreported ways which differ depending on whether the spin-down mole fraction $X_{j}$ increases, decreases, or remains constant with increasing $N$. Because these $X_{j}$-dependencies are monotonic in $N$, PSL-based nanothermometers can in principle provide meaningful temperature estimates for systems containing fewer than $10^{3}$ particles.

Second, although the four Boltzmann ESTs of PSLs are not true thermodynamic temperatures, they are useful temperature estimators for the full eigenstate spectrum of small PSLs. Gibbs ESTs are also useful temperature estimators-but only for positive temperature eigenstates; for population-inverted eigenstates, Boltzmann ESTs provide reliable temperature estimates, whereas the Gibbs ESTs do not.

Third, the thermodynamic uncertainty relation ${ }^{34,35}$ is violated by microcanonical PSLs of any size and by large canonical PSLs in equilibrium with infinite baths; it is obeyed only by finite canonical PSLs in equilibrium with infinite baths. ${ }^{34-36,67,105,106,126}$ Temperature measurements are thus non-fluctuating in microcanonical PSLs of any size, nonfluctuating and thermodynamically accurate in large canonical PSLs, ${ }^{42,70-73,126}$ and fluctuating and thermodynamically approximate in finite canonical PSLs.

Fourth, intensity of temperature is a more important criterion for genuine thermodynamic behavior than adiabatic invariance of the entropy. ${ }^{5,133}$

Collectively, our results suggest that ESTs will provide insights into a number of important current topics, including (1) the relative impacts of finite-size effects ${ }^{36,40,60,68,83,84,92,93}$ and long-range interactions ${ }^{36,60-62,66-68,74,83,84,92-94,103-106}$ on thermostatistical behavior, (2) the eigenstate thermalization hypothesis, ${ }^{138,139}$ (3) the potential impact of temperature-dependent system energy levels on the thermodynamic uncertainty relation, ${ }^{33,121,129}$ and (4) nanothermometry. ${ }^{60,83,84,90-102}$

\section{SUPPLEMENTARY MATERIAL}

See supplementary material for details regarding (A) the spin-permutation antisymmetries of the ESTs ${ }^{119}$ and (B) the impact of spin-down mole fraction $X_{j}$ on the continuous microcanonical, discrete canonical, and discrete microcanonical ESTs of constant- $X_{j}$, increasing- $X_{j}$, and decreasing- $X_{j}$ eigenstates. ${ }^{120,121}$

\section{ACKNOWLEDGMENTS}

The authors thank R. Ananthoji and R. N. Karingithi for contributions to this research during its earliest stages, Mr. T. J. Masthay, Mr. T. M. Masthay, Dr. J. E. Adams, Dr. V. A. Benin, Dr. R. L. Berney, Dr. C. J. Cairns, Dr. G. S. Crosson, Dr. R. Lustig, Dr. P. G. Nelson, Dr. J. M. Standard, and Dr. M. Usman for helpful discussions, and J. T. Allen, D. A. Bucher, M. E. Griffin, M. E. Kelleher, A. Khan, J. M. Mabrouk, 
K. P. Mayrand, J. B. McGregor, T. Pair, R. J. Provost, R. G. Raspberry, M. L. Rudolph, J. A. Schatz, M. R. Short, T. S. Sirls, and L. K. White for editorial assistance. M.B.M. thanks the National Science Foundation (No. NSF-EPS-0132295) and the Howard Hughes Medical Institute (Undergraduate Biological Sciences Education Initiative Year 2000 Award) for partial funding of this research.

${ }^{1}$ H. Feshbach, Phys. Today 40(11), 9 (1987).

${ }^{2}$ C. Kittel, Phys. Today 41(5), 93 (1988).

${ }^{3}$ B. B. Mandelbrot, Phys. Today 42(1), 71 (1989).

${ }^{4}$ J. Dunkel and S. Hilbert, Nat. Phys. 10, 67 (2013).

5 J. M. G. Vilar and J. M. Rubi, J. Chem. Phys. 140, 201101 (2014).

${ }^{6}$ D. Frenkel and P. B. Warren, Am. J. Phys. 83, 163 (2015).

${ }^{7}$ N. F. Ramsey, in A Critical Review of Thermodynamics, edited by E. B. Stuart, A. J. Brainard, and B. Gal-Or (Mono Book Corporation, Baltimore, 1970), p. 217.

${ }^{8}$ B. H. Lavenda, J. Phys. A: Math. Gen. 32, 4279 (1999).

${ }^{9}$ J. Wisniak, J. Chem. Educ. 77, 518 (2000).

${ }^{10}$ See pp. 34-35, 48-49, and 320-323 of F. Schwabl, Statistical Mechanics (Springer-Verlag, Berlin-Heidelberg, 2006), Tr. W. Brewer.

${ }^{11}$ See pp. 89-96 of C. Kittel, Thermal Physics (John Wiley \& Sons, Inc., New York, NY, 1969).

${ }^{12}$ M. B. Masthay and H. B. Fannin, J. Chem. Educ. 82, 867 (2005).

${ }^{13}$ N. F. Ramsey and R. V. Pound, Phys. Rev. 81, 278 (1951).

${ }^{14}$ R. V. Pound, Phys. Rev. 81, 156 (1951).

${ }^{15}$ E. M. Purcell and R. V. Pound, Phys. Rev. 81, 279 (1951).

${ }^{16}$ A. Abragam and W. G. Proctor, Phys. Rev. 106, 160 (1957).

${ }^{17}$ A. Abragam and W. G. Proctor, Phys. Rev. 109, 1441 (1958).

${ }^{18}$ See pp. 211-214 of L. D. Landau and E. M. Lifschitz, Course of Theoretical Physics Volume 5: Statistical Physics, 2nd ed. (Addison-Wesley, Reading, MA, 1969).

${ }^{19}$ G. Bhanot and M. Creutz, Phys. Rev. B 22, 3370 (1980).

${ }^{20}$ C. E. Hecht, J. Chem. Educ. 44, 124 (1967).

${ }^{21}$ A. A. Popov, V. V. Sherstnev, and Z. Zelinger, Tech. Phys. Lett. 23, 890 (1997).

${ }^{22}$ M. Goldman, Appl. Magn. Reson. 34, 219 (2008).

${ }^{23}$ Y. D. Kalafati and D. V. Posvyanskii, JETP Lett. 57, 676 (1993).

${ }^{24}$ Y. A. Byovskii, I. A. Farifullin, V. F. Elesin, V. I. Kadushkin, E. A. Protasov, and A. G. Radionov, Sov. Phys. J. 15, 230 (1972).

${ }^{25}$ G. L. Eyink and H. Spohn, J. Stat. Phys. 70, 833 (1993).

${ }^{26}$ L. Onsager, Il Nuovo Cimento 6, 279 (1949).

${ }^{27}$ V. Berdichevsky, I. Kunin, and F. Hussain, Phys. Rev. A 43, 2050 (1991).

${ }^{28}$ S. Braun, J. P. Ronzheimer, M. Schreiber, S. S. Hodgman, T. Rom, I. Bloch, and U. Schneider, Science 339, 52 (2013).

${ }^{29}$ A. Rapp, S. Mandt, and A. Rosch, Phys. Rev. Lett. 105, 220405 (2010).

${ }^{30}$ A. P. Mosk, Phys. Rev. Lett. 95, 040403 (2005).

${ }^{31}$ U. Schneider, S. Mandt, A. Rapp, S. Braun, H. Weimer, I. Bloch, and A. Rosch, e-print arXiv:1407.4127v1 [cond-mat.quant-gas] (2014).

${ }^{32}$ P. Medley, D. M. Weld, H. P. Miyake, D. E. Pritchard, and W. Ketterle, Phys. Rev. Lett. 106, 195301 (2011).

${ }^{33}$ R. de Miguel and J. M. Rubi, J. Phys. Chem. B 120, 9180 (2016).

${ }^{34}$ J. Uffink and J. van Lith, Found. Phys. 29, 655 (1999).

${ }^{35}$ L. Velazquez and S. Curilef, Mod. Phys. Lett. B 23, 3551 (2009).

${ }^{36}$ T. Jahnke, S. Lanéry, and G. Mahler, Phys. Rev. E 83, 011109 (2011).

${ }^{37}$ E. D. Belega, E. A. Cheremukhin, P. V. Elyutin, and D. N. Trubnikov, Chem. Phys. Lett. 496, 167 (2010).

${ }^{38}$ A. A. Caparica and C. J. DaSilva, e-print arXiv 1112.1907v4 [condmat.stat-mech] (2015).

${ }^{39}$ R. Franzosi, J. Stat. Phys. 143, 824 (2011).

${ }^{40}$ J. Gemmer, M. Michel, and G. Mahler, Quantum Thermodynamics: Emergence of Thermodynamic Behavior within Composite Quantum Systems, Lecture Notes in Physics, 2nd ed. (Springer-Verlag, Heidelberg, 2009), Vol. 784.

${ }^{41}$ M. J. Davis and P. B. Blakie, J. Phys. A: Math. Gen. 38, 10259 (2005).

${ }^{42} \mathrm{H}$. Touchette, in Nonextensive Entropy: Interdisciplinary Applications, edited by M. Gell-Mann and C. Tsallis (Oxford University Press, New York, 2004), p. 159.

${ }^{43}$ R. B. Shirts and M. R. Shirts, J. Chem. Phys. 117, 5564 (2002).

${ }^{44}$ U. Salian, J. Chem. Phys. 108, 6342 (1998).

${ }^{45}$ K. Takatsuka and T. Yanao, J. Chem. Phys. 113, 2552 (2000).

${ }^{46}$ H. H. Rugh, J. Phys. A: Math. Gen. 31, 7761 (1998).
${ }^{47}$ G. Rickayzen and J. G. Powles, J. Chem. Phys. 114, 4333 (2001).

${ }^{48}$ B. D. Butler, G. Ayton, O. G. Jepps, and D. J. Evans, J. Chem. Phys. 109, 6519 (1998).

${ }^{49}$ O. G. Jepps, G. Ayton, and D. J. Evans, Phys. Rev. E 62, 4757 (2000).

${ }^{50}$ A. Baranyai, J. Chem. Phys. 112, 3964 (2000).

${ }^{51}$ K. C. Chase, A. Z. Mekjian, and I. Zamick, Eur. Phys. J. B 8, 281 (1999).

${ }^{52}$ G. Ayton, O. G. Jepps, and D. J. Evans, Mol. Phys. 96, 915 (1999).

${ }^{53}$ G. P. Morriss and L. Rondoni, Phys. Rev. E 59, R5 (1999).

${ }^{54}$ C. Dress, J. Phys. A: Math. Gen. 28, 7051 (1995).

${ }^{55}$ C. Giardinà and R. Livi, J. Stat. Phys. 91, 1027 (1998).

${ }^{56}$ L. Lue and D. J. Evans, Phys. Rev. E 62, 4764 (2000).

${ }^{57}$ J. U. Andersen, E. Bonderup, and K. Hansen, J. Chem. Phys. 114, 6518 (2001).

${ }^{58}$ M. Schmidt, R. Kusche, T. Hippler, J. Donges, W. Kronmüller, B. von Issendorf, and H. Haberland, Phys. Rev. Lett. 86, 1191 (2001).

${ }^{59}$ R. C. Dunbar, J. Chem. Phys. 90, 7369 (1989).

${ }^{60}$ M. Hartmann and G. Mahler, Europhys. Lett. 70, 579 (2005).

${ }^{61}$ L. C. Sampaio, M. P. de Albuquerque, and F. S. de Menezes, Phys. Rev. B 55, 5611 (1997).

${ }^{62}$ F. D. Nobre and C. Tsallis, Philos. Mag. B 73, 745 (1996).

${ }^{63}$ J. Lee, J. Chem. Educ. 42, 340 (1965).

${ }^{64}$ P. Hakonen and O. V. Lounasmaa, Science 265, 1821 (1994).

${ }^{65}$ R. T. Vuorinen, P. J. Hakonen, W. Yao, and O. V. Lounasmaa, J. Low Temp. Phys. 98, 449 (1995).

${ }^{66}$ F. Caruso and C. Tsallis, AIP Conf. Proc. 965, 51 (2007).

${ }^{67}$ F. Pennini, A. Plastino, A. R. Plastino, and M. Casas, Phys. Lett. A 302, 156 (2002).

${ }^{68}$ T. Jahnke and G. Mahler, Phys. Rev. E 84, 011129 (2011).

${ }^{69} \mathrm{~J}$. Lindhard, in The Lesson of Quantum Theory, edited by J. de Boer, E. Dal, and O. Ulfbeck (North Holland, New York, 1986).

${ }^{70}$ H. Touchette, Am. J. Phys. 76, 26 (2008).

${ }^{71} \mathrm{H}$. Touchette, R. S. Ellis, and B. Turkington, Phys. A 340, 138 (2004).

72" For the average square of the anomalies of the energy, we find an expression which vanishes in comparison to the square of the average energy, when the number of degrees of freedom is indefinitely increased. An ensemble of systems in which the number of degrees of freedom is of the same order of magnitude as the number of molecules in the bodies with which we experiment, if distributed canonically, would therefore appear to human observation as an ensemble of systems in which all have the same energy." (p. xi of J. W. Gibbs, Elementary Principles in Statistical Mechanics with Especial Reference to the Rational Foundation of Thermodynamics (Yale University Press, Yale, CT, 1902), reprinted by Dover, New York, 1960.

${ }^{73}$ See pp. 10-12, 27-29, and (especially) 40-42 of T. L. Hill, An Introduction to Statistical Thermodynamics (Addison-Wesley, Reading, MA, 1960) and pp. 63-64 of D. A. McQuarrie, Statistical Mechanics (Harper \& Row, New York, NY, 1976).

${ }^{74}$ T. Jahnke and G. Mahler, Europhys. Lett. 90, 50008 (2010).

${ }^{75}$ H. Dong, X. F. Liu, and C. P. Sun, Chin. Sci. Bull. 55, 3256 (2010).

${ }^{76}$ G. Gordon, N. Erez, and G. Kurizki, Opt. Spectrosc. 108, 400 (2010).

${ }^{77}$ G. A. Álvarez, D. D. Bhaktavatsala Rao, L. Frydman, and G. Kurizki, Phys. Rev. Lett. 105, 160401 (2010).

${ }^{78}$ Scenario (iv) gives rise to the SEOC for the $[N / 2, N / 2]$ and $[N, 0]$ eigenstates of finite PSLs in contact with heat baths; it has not been shown to give rise to the SEOC for other eigenstates (see Refs. 36, 68, and 74-77). Hence, like scenario (ii), scenario (iv) may be more restricted in its ability to give rise to the SEOC than scenarios (i) and (iii), which can in principle give rise to the SEOC for any PSL eigenstate.

${ }^{79} \mathrm{~N}$. Bloembergen, Physica 15, 386 (1949).

${ }^{80}$ N. Bloembergen and T. J. Rowland, Phys. Rev. 97, 1679 (1955).

${ }^{81}$ B. V. Rollin, Rep. Prog. Phys. 12, 22 (1949).

${ }^{82}$ L. G. Rizzi and N. A. Alves, J. Chem. Phys. 135, 141101 (2011).

${ }^{83}$ M. Hartmann, G. Mahler, and O. Hess, Phys. E 29, 66 (2005).

${ }^{84}$ M. Hartmann, G. Mahler, and O. Hess, Phys. Rev. E 70, 066148 (2004).

${ }^{85}$ M. Kastner, M. Promberger, and A. Hüller, in Computer Simulational Studies in Condensed Matter Physics XI, edited by D. P. Landau and H.-B. Schüttler (Springer-Verlag, Heidelberg, 1999), p. 161.

${ }^{86}$ S. M. Aldoshin, J. Photochem. Photobiol., A 200, 19 (2008).

${ }^{87}$ See pp. $245-385$ of P. W. Selwood, Magnetochemistry, 2nd ed. (Interscience Publishers, New York, NY, 1956).

${ }^{88}$ Y. A. Freiman and H. J. Jodl, Phys. Rep. 401, 1 (2004).

${ }^{89}$ M. S. Reis, J. C. C. Freitas, M. T. D. Orlando, E. K. Lenzi, and I. S. Oliveira, Europhys. Lett. 58, 42 (2002).

${ }^{90}$ F. Seilmeier, M. Hauck, E. Schubert, G. J. Schinner, S. E. Beavan, and A. Högele, Phys. Rev. Appl. 2, 024002 (2014). 
${ }^{91}$ F. Haupt, A. Imamoglu, and M. Kroner, Phys. Rev. Appl. 2, 024001 (2014).

${ }^{92}$ M. Hartmann, G. Mahler, and O. Hess, J. Phys. Soc. Jpn. 74, 26 (2005).

${ }^{93}$ M. Hartmann, G. Mahler, and O. Hess, Phys. Rev. Lett. 93, 080402 (2004).

${ }^{94}$ M. Hartmann, J. Gemmer, G. Mahler, and O. Hess, Europhys. Lett. 65, 613 (2004).

${ }^{95}$ T. C. P. Chui, D. R. Swanson, M. J. Adriaans, J. A. Nissen, and J. A. Lipa, Phys. Rev. Lett. 69, 3005 (1992).

${ }^{96}$ Q. Dai, Y. Zhang, Y. Wang, M. Z. Hu, B. Zou, Y. Wang, and W. W. Yu, Langmuir 26, 11435 (2010).

${ }^{97}$ P. Neumann, I. Jakobi, F. Dolde, C. Burk, R. Reuter, G. Waldherr, J. Honert, T. Wolf, A. Brunner, J. H. Shim, D. Suter, H. Sumiya, J. Isoya, and J. Wrachtrup, Nano Lett. 13, 2738 (2013).

${ }^{98}$ Q. Xiang and P. C. Morais, Nanomed. Nanotechnol. 5, 1000209 (2014).

${ }^{99}$ J. B. Weaver, J. Appl. Phys. 111, 07B317 (2012).

${ }^{100}$ J. B. Weaver, A. M. Rauwerdink, and E. W. Hanson, Med. Phys. 36, 1822 (2009).

${ }^{101}$ A. M. Rauwerdink, E. W. Hanson, and J. B. Weaver, Phys. Med. Biol. 54, L51 (2009).

${ }^{102}$ J. Zhong, W. Liu, Z. Du, P. C. Morais, Q. Xizng, and Q. Xie, Nanotechnology 23, 075703 (2012).

${ }^{103}$ C. Tsallis, A. Rapisarda, A. Pluchino, and E. P. Borges, Phys. A 381, 143 (2007).

${ }^{104}$ V. Latora and A. Rapisarda, in Nonextensive Entropy: Interdisciplinary Applications, edited by M. Gell-Mann and C. Tsallis (Oxford University Press, New York, 2004), p. 113.

${ }^{105}$ A. R. Plastino and A. Plastino, Phys. Lett. A 193, 140 (1994).

${ }^{106}$ M. Baranger, Phys. A 305, 27 (2001).

${ }^{107}$ See p. 72 of A. F. Orchard, Magnetochemistry (Oxford University Press, Oxford, UK, 2003).

${ }^{108}$ S. Elliott, The Physics and Chemistry of Solids (John Wiley \& Sons, Chichester, 1998).

${ }^{109}$ See pp. 140-148, 185, 264 of J. Gemmer, M. Michel, and G. Mahler, Quantum Thermodynamics: Emergence of Thermodynamic Behavior Within Composite Quantum Systems, 2nd ed. (Springer-Verlag, Heidelberg, 2009).

${ }^{110}$ See p. 400 of C. Kittel, Introduction to Solid State Physics, 6th ed. (John Wiley \& Sons, Inc., New York, NY, 1986).

${ }^{111}$ N. F. Ramsey, Phys. Rev. 103, 20 (1956).

${ }^{112}$ See pp. $85-92,97$ of R. K. Pathria, Statistical Mechanics (Pergamon, Oxford, 1972).

${ }^{113}[N+1,-1] \rightarrow[N, 0]$ and $[0, N] \rightarrow[-1, N+1]$ transitions are not physically realistic because $[N+1,-1]$ and $[-1, N+1]$ are not PSL eigenstates. These transitions are accordingly not discussed in the text, though $T_{c}{ }^{c o n v}, T_{c}{ }^{j \rightarrow j+1}$, $T_{\mu}{ }^{j \rightarrow j+1}$, and $T_{d c}{ }^{j \rightarrow j+1}$ are mathematically well behaved for $j=-1$ and $N$, as is $T_{d \mu}{ }^{j \rightarrow j+1}$ for $j=N$.

${ }^{114}$ Though an infinite number of analytic functions pass through the factorials of all positive integers, $\Gamma(x)$ is the only function which is uniquely determined for all positive real numbers because - in accord with the BohrMollerup Theorem-it satisfies the conditions $\Gamma(1)=1, \Gamma(x+1)=x \Gamma(x)$ and is logarithmically convex. The gamma function is thus the only reasonable analytic function for representing factorials, see the work of Artin and Emil, "The Gamma Function" in M. Rosen, Exposition by Emil Artin: A Selection, History of Mathematics 30 (American Mathematical Society, Providence, RI, 2006).

${ }^{115} \mathrm{H}$. Jeffreys and B. S. Jeffreys, Methods in Mathematical Physics, 3rd ed. (Cambridge University Press, Cambridge, 1988), p. 465.

${ }^{116}$ The infinite temperature asymptotes of the discrete $j=N / 2 \rightarrow(N / 2+1)$ ESTs are displaced by $\Delta_{j}$, asymptote $=-0.5$ and $\Delta X_{j, \text { asymptote }}=-0.5 / \mathrm{N}$ from those of the continuous $j=N / 2 \rightarrow(N / 2+1)$ ESTs.

${ }^{117} T_{d c}^{j \rightarrow j+1}$ is imaginary for $j=N$, since the argument of the logarithm is negative and $\ln (-1)=i \pi$

${ }^{118}$ See pp. $10-12$ of D. A. McQuarrie, Statistical Mechanics (University Science Books, Sausalito, CA, 2000).

${ }^{119}$ de Miguel and Rubi obtain a different—but related — set of spin permutation antisymmetries for the discrete microcanonical ESTs $T_{d \mu}{ }^{j-1 \rightarrow j}$ defined in terms of "backward" $j-1 \rightarrow j$ transitions. Our four ESTs are all defined in terms of "forward" $j \rightarrow j+1$ transitions ["forward" and "backward" terminology taken from Ref. 33 and from T. Wada and A. M. Scarfone, J. Phys.: Conf. Ser. 201, 0125 (2010)].

${ }^{120} \mathrm{The} X_{j}$-dependence of our forward $\left(T_{d \mu}{ }^{j \rightarrow j+1}\right)$ and de Miguel and Rubi's backward $\left(T_{d \mu}{ }^{j-1 \rightarrow j}\right)$ discrete microcanonical ESTs (see Refs. 33 and 119) are opposite to each other. For example, the values of $T_{d \mu}{ }^{j \rightarrow j+1}$ are large for small $N$ and fall to their thermodynamic limiting values near $N=1000$ [see Fig. 3(c) in the revised manuscript], whereas the values of $T_{d \mu}{ }^{j-1 \rightarrow j}$ are small for small $N$ and rise to their intensive, thermodynamic limiting values near $N=1000$ (from our plots of de Miguel and Rubi's $T_{d \mu}{ }^{j-1 \rightarrow j}$ values; data are not shown).

${ }^{121}$ The presence of the functional $X_{j}$-dependencies in both forward and backward discrete microcanonical ESTs (see Refs. 33 and 119) precludes the possibility that these $X_{j}$-dependencies originate from temperaturedependent system energy levels (TDSELs), since the Hamiltonians of thermally isolated systems are not perturbed by bath Hamiltonians.

${ }^{122}$ The continuous canonical ESTs for constant- $X_{j}$ eigenstates-which are inherently intensive-are the single exception to this rule.

${ }^{123}$ See Eq. (34.7) and surrounding discussion in R. Becker, Theory of Heat, 2nd ed. (Springer-Verlag, New York, NY, 1967).

${ }^{124}$ See pp. $186-190$ of J. R. Waldram, The Theory of Thermodynamics (Cambridge University Press, Cambridge, 1985).

${ }^{125}$ Using backward $j=-1 \rightarrow j=0$ transitions (see Refs. 33 and 119), de Miguel and Rubi find that the discrete microcanonical temperature of the perfect crystalline (i.e., all-spins-down) PSL ground state $[N, 0]$ is equal to zero: $T_{d \mu}{ }^{\mathrm{j}-1 \rightarrow \mathrm{j}}=T_{d \mu}{ }^{-1 \rightarrow 0}=0$, in accord with the third law. However, to obtain this result, they must assume that the ground state temperature is equal to that of a transition originating from a non-physical $[N+1,-1]$ "eigenstate" lying below the ground eigenstate (see Ref. 113). In contrast, we assume that the ground state temperature $T_{d \mu}{ }^{\mathrm{j} \rightarrow \mathrm{j}+1}=T_{d \mu}{ }^{0 \rightarrow 1}$ is equal to that of a forward $j=0$ $\rightarrow j=1$ transition between the two genuine, physical PSL eigenstates $[N, 0]$ and $[N-1,1]$. While the forward continuous canonical EST $T_{c}^{0 \rightarrow 1}$ is equal to zero for all $N$, the forward ESTs $T_{d \mu}{ }^{0 \rightarrow 1}, T_{\mu}{ }^{0 \rightarrow 1}$, and $T_{d c}{ }^{0 \rightarrow 1}$ are all greater than zero for finite $N$ and approach zero in the limit of large $N$. Our results thus indicate that for the ground state in PSLs, forward ESTs are consistent with the third law-but generally only in the TDL. It is interesting to note that for $j=N \rightarrow j=N+1$ transitions from the perfect crystalline (i.e., all-spins-down), maximum energy eigenstate $[0, N]$ to the non-physical state $[-1, N+1]$ directly above it (see Ref. 113), backward $T_{d \mu}{ }^{j-1 \rightarrow j}$ values are finite and negative and approach -0 in the thermodynamic limit, whereas forward $T_{d \mu}{ }^{j \rightarrow j+1}$ values are equal to -0 for all $N$; i.e., there is a symmetry between the $N$-dependence of $T_{d \mu}{ }^{j-1 \rightarrow j}$ and $T_{d \mu}{ }^{j \rightarrow j+1}$ for the $[N, 0]$ and $[0, N]$ eigenstates, which suggests a difference in zero-point energy for backward and forward discrete microcanonical ESTs. This symmetry is related to the spin permutation antisymmetries and may be rooted in the differences in the second law for negative and positive temperature eigenstates proposed by Lavenda (see Sec. III B, the supplementary material, and Ref. 8).

${ }^{126}$ Boltzmann statistics apply only to systems in equilibrium with baths which are infinite $\left(N_{\text {bath }} \rightarrow \infty\right)$, energetically quasi-continuous $(\varepsilon \rightarrow 0)$, and which (generally) lack long-range interactions. For systems in contact with baths which do not satisfy these conditions, Tsallis statistics prevail (see Refs. 67, 105, and 106). Since the TUR assumes Boltzmann statistics, it applies only to finite canonical PSLs in equilibrium with infinite baths (see Refs. 34 and 106).

${ }^{127}$ This disagreement does not negate the importance of Mandelbrot's contributions to TUR research for two reasons. First, his results regarding the applicability of the TUR to canonical systems agree with both our results and those of Uffink and van Lith (see Ref. 34). Second, in assuming that a single eigenstate extracted from a Boltzmann distribution retains the thermostatistical content of the full distribution, Mandelbrot appears to have anticipated the eigenstate thermalization hypothesis; see Refs. 138 and 139.

${ }^{128}$ In spite of his mistaken contention that the TUR applies to finite microcanonical systems, Mandelbrot was aware that the TUR fails in the TDL, for which he noted that "temperature fluctuations ... are negligibly small" (see Ref. 3). This raises the following question: "Did Mandelbrot incorrectly conflate the $N \rightarrow \infty$ requirement for a single microcanonical EST to be thermodynamically accurate with the requirements for the TUR to fail?" The answer appears to be no, as Mandelbrot based his arguments on the "zeroth principal of thermodynamics"- not on large $N$ behavior.

${ }^{129}$ When there is a mismatch between energy level spacings of small systems and their heat baths, small canonical systems are able to equilibrate with their heat baths only when the system energy levels are modulated by interaction with the bath (i.e., when TDSELs are operative). In such cases, the mismatch between $T_{\text {system }}$ and $T_{\text {bath }}$ "drives a process that produces an amount of work which is used to perturb the quantum spectrum of the finite system. It is this temperature-dependent spectrum perturbation 
which makes possible the thermal equilibrium between the finite system and the heat bath" (quoted from Ref. 33). This scenario, in combination with the fact that both TDSELs and the TUR by definition apply only to finite canonical systems, raises an interesting question: "Since the energy levels of small canonical systems are perturbed by their baths, do TDSELs contribute to the energy and temperature uncertainties associated with the TUR in such systems?" (see Refs. 34, 67, 105, 106, and 126). This suggestion seems plausible particularly because the TUR does not apply to microcanonical (i.e., thermally isolated) systems (see Ref. 121). TDSELs may also play a role in the generation of negative temperature eigenstates in PSLs which are out of "resonance" with their baths $\left(\varepsilon_{\mathrm{PSL}} \neq \varepsilon_{\text {bath }}\right.$; see Ref. 68).

${ }^{130}$ M. Kliesch, C. Gogolin, M. J. Kastoryano, A. Riera, and J. Eisert, Phys. Rev. X 4, 031109 (2014)

${ }^{131}$ S. Hernández-Santana, A. Riera, K. V. Hovhannisyan, M. Perarnau-Llobet, L. Tagliacozzo, and A. Acín, New J. Phys. 17, 085007 (2015).

${ }^{132}$ P. Hertz, Ann. Phys. 338, 225 (1910).
${ }^{133}$ A. Tavassoli and A. Montakhab, e-print arXiv:1512.02855v2 [cond-mat.stat-mech] (2015).

${ }^{134}$ A population-inverted $\left(X_{1 / 2}=0.50000036\right.$ and $X_{3 / 2}=0.49999964$, with $\left.\varepsilon=U_{1 / 2}-U_{3 / 2}=6.99 \times 10^{-27} \mathrm{~J}\right)$ collection of $10^{21}{ }^{7} \mathrm{Li}$ nuclei in the earth's $0.6376 \mathrm{~T}$ field manifested a nuclear spin temperature of $-350 \mathrm{~K}$ (see Ref. 15). As $N=10^{21}$, each of the four ESTs were converged to their thermodynamic limiting value of $-350 \mathrm{~K}$, corresponding to $j=5.0000036$ $\times 10^{20} \rightarrow\left(5.0000036 \times 10^{20}\right)+1$ transitions

${ }^{135}$ J. P. Bergfield, S. M. Story, R. C. Stafford, and C. A. Stafford, ACS Nano 7, 4429 (2013).

${ }^{136}$ J. Meair, J. P. Bergfield, C. A. Stafford, and P. Jacquod, Phys. Rev. B 90, 035407 (2014).

${ }^{137}$ Jahnke and Mahler derive an expression for $T_{\mu}{ }^{j \rightarrow j+1}$ [identical to our Eq. (8a)] but apply it only under specialized conditions in which it is equivalent to $T_{c}{ }^{j \rightarrow j+1}$ see their Eq. (3) and Appendix B.

${ }^{138}$ M. Rigol and M. Srednicki, Phys. Rev. Lett. 108, 110601 (2012).

${ }^{139}$ J. M. Deutsch, New J. Phys. 12, 075021 (2010). 